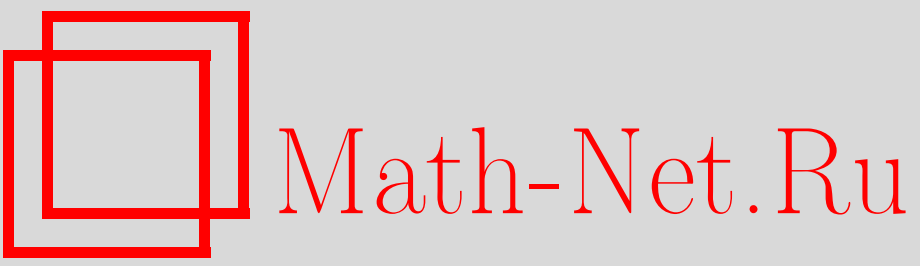

К. А. Свешников, П. К. Силаев, О взаимосвязи между разрывными и гладкими решениями типа кинков в квантовой теории поля, ТМФ, 1996, том 108, номер 2, 212-248

DOI: https://doi.org/10.4213/tmf1188

Использование Общероссийского математического портала Math-Net.Ru подразумевает, что вы прочитали и согласны с пользовательским соглашением

http: //www . mathnet.ru/rus/agreement

Параметры загрузки:

IP : 3.85 .183 .62

26 апреля 2023 г., 16:54:12 
ТЕОРЕТИЧЕСКАЯ

И МАТЕМАТИЧЕСКАЯ

ФИЗИКА

Том 108, № 2

август, 1996

К. А. Свешников, П. К. Силаев

\title{
О ВЗАИМОСВЯЗИ МЕЖДУ РАЗРЫВНЫМИ И ГЛАДКИМИ РЕШЕНИЯМИ ТИПА КИНКОВ В КВАНТОВОЙ ТЕОРИИ ПОЛЯ
}

\begin{abstract}
Предлагается непертурбативная процедура вычитания расходимостей, обусловленных наличием конечных разрывов полевой конфигурации. В качестве приложения рассмотрено лоренц-ковариантное квантование 1+1-мерных топологических кинков. Показано, что при этом естественным образом возникают "квантовые копии" классического кинка, которые имеют такую же топологию, но исчезающе малые размеры и массу, т.к. вычитание уничтожает расходимости, возникаюшие при дифференцировании поля в точках разрыва. Эти эффекты подробно исследованы на примере $(1+1)$-мерных моделей скалярного поля, в которых существуют классические кинки.
\end{abstract}

\section{1. ВВЕДЕНИЕ}

В последнее время все большее внимание привлекают к себе частицеподобные классические решения в существенно нелинейных моделях квантовой теории поля как возможный способ описания квантовых протяженных объектов типа барионов [1-3]. Как правило, такое классическое решение $\varphi_{c}(x)$ интерполирует между статическими вакуумными значениями поля, которые соответствуют минимумам (эффективного) полевого потенциала. Поэтому для каждого такого решения всегда можно построить топологически идентичное разрывное кусочно-постоянное решение $\varphi_{d}(x)$, которое отличается от непрерывного только тем, что размер переходной области равен нулю. Так, в $(1+1)$-мерном случае типичное кинковое решение имеет вид $\varphi_{c}(x)=$ th $x$, его разрывным аналогом является $\varphi_{d}(x)=\operatorname{sign} x$. Чтобы построить разрывной аналог для $3+1$-мерных скирмионных конфигураций, достаточно заменить киральный угол $F(r)$, который непрерывно меняется от $F(0)=\pi n$ до $F(\infty)=0$, функцией $F_{d}(r)=\pi n \theta(R-r)$.

До настояшего времени такие разрьвные решения не вызывали особого интереса, поскольку их классическая энергия бесконечна из-за квадрата $\delta$-функции, возникаюшего из "кинетического" члена в гамильтониане. Однако при ближайшем рассмотрении ситуация с разрывными решениями оказывается не столь тривиальной. Действительно, в данном случае природа расходимостей состоит в том, что для разрывного решения высокочастотная составляюшая поля недостаточно быстро убывает при $|k| \rightarrow \infty$, и тем самым такие расходимости имеют явно ультрафиолетовый характер. В то же время 
в перенормируемой теории все ультрафиолетовые расходимости устраняются соответствующей регуляризацией с последующим вычитанием. Поэтому напрашивается естественное предположение, что при разумной вычитательной процедуре в квантованном случае должно происходить сглаживание сингулярностей классической теории, обусловленных конечными разрывами.

В настояшей работе мы приведем аргументы в пользу такого утверждения, рассмотрев в качестве примера ковариантное квантование 1+1-мерных кинков с помошью релятивистских операторов координат Ньютона-Вигнера. Исходным пунктом такого подхода является обшее условие ковариантности теории относительно группы Пуанкаре, которое гласит, что при преобразованиях этой группы $x \rightarrow x^{\prime}=\Lambda x+a$ любое квантовое поле должно преобразовываться следуюшим образом:

$$
\varphi(x) \rightarrow \varphi^{\prime}(x)=U^{+}(\Lambda, a) \varphi(x) U(\Lambda, a)=S(\Lambda) \varphi\left(\Lambda^{-1}(x-a)\right),
$$

где $S(\Lambda)$ - соответствуюшее конечномерное представление группы Лоренца.

Хорошо известно, что при квантовании фолуктуаций поля в окрестности одного из минимумов потенциала, т.е. в отсутствие нетривиального классического решения, теория оказывается автоматически совместной с условием (1.1), если в качестве генераторов унитарных преобразований в (1.1) использовать соответствующие нетеровские интегралы движения. Однако, если попытаться аналогичным образом квантовать в кинковом секторе, мы столкнемся с тем, что кинковая составляющая поля, с одной стороны, при преобразованиях группы Пуанкаре должна изменяться, как это предписывается уравнением (1.1), а с другой стороны, являясь $c$-числом, она коммутирует со всеми генераторами группы Пуанкаре, построенными с помошью теоремы Нетер. Таким образом, наличие $c$-числового решения от $c$-числового аргумента явным образом нарушает ковариантность относительно группы Пуанкаре. Единственный способ восстановить ковариантность заключается в том, чтобы таким образом провести разложение поля на кинковую и мезонную части:

$$
\varphi(x)=\varphi_{K}(x)+\Phi(x)
$$

чтобы условие ковариантности (1.1) выполнялось по отдельности для каждой из частей равенства (1.2). Другими словами, кинковая составляющая $\varphi_{K}(x)$ должна быть сама по себе ковариантным оператором. В противном случае в спектре возмущений возникнут нулевые моды с линейной по времени, а не с осциллирующей динамикой, которые в некотором смысле являются компенсацией нековариантности $c$-числового решения. Нулевые моды, разумеется, невозможно рассматривать как "малые" возмущения, поэтому все разложение на мезонную и кинковую части также становится невозможным.

Чтобы обеспечить явную ковариантность кинковой компоненты, мы должны ввести в аргумент поля соответствуюшие групповые координаты, которые восстановят пуанкаре-инвариантность и обеспечат исчезновение нулевых мод. Эти координаты являются квантово-полевыми аналогами релятивистских операторов координат Ньютона-Вигнера [4] и на самом деле естественным образом возникают в процессе суммирования секулярных членов ряда теории возмушений, рассматриваемой в окрестности 
классического решения [5], поскольку секулярные члены соответствуют смешению кинка как целого и поэтому эффективно эквивалентны введению ковариантных групповых переменных.

Результатом появления переменных Ньютона-Вигнера в динамической схеме квантованной теории является преврашение всех дифференциальных операторов в уравнениях движения в операторы конечных разностей, шаг в которых пропорционален эффективной постоянной Планка $\hbar$ теории [6]. При этом автоматически происходит выделение сингулярностей, связанных с дифференцированием конечных разрывов, в виде специфических полюсных членов. Эти полюсные члены могут быть представлены в форме, вполне аналогичной стандартным ультрафиолетовым расходимостям квантовой теории, в частности энергии нулевых колебаний, записанной через быстротное представление. Постулируя вычитание таких полюсных членов, мы получим вполне содержательную процедуру ковариантного квантования кинков, в которой конечные разрывы полевой конфигурации не дают расходимостей, а напротив, их энергия убывает с уменьшением переходной области разрыва. Последний результат является прямым следствием того, что такое вычитание эффективно эквивалентно некоторому непертурбативному ультрафиолетовому обрезанию теории. Действительно, при уменьшении переходной области относительный вес высокочастотной составляющей поля возрастает и она дает главный (расходящийся) вклад во все наблюдаемые эффекты, который и уничтожается вычитанием. При этом существенно, что конечный произвол в такой процедуре вычитания расходящихся величин может быть однозначно зафиксирован требованием неизменности энергии и других свойств гладких (без разрывов) полевых конфигураций.

Как следствие при таком вычитании теория допускает, помимо исходного классического кинка, сушествование еще и его “квантовых копий”, топологически идентичных исходному решению, но с массой, пропорциональной $\sqrt{\hbar / N}[6]$, где $N$ - целое. При этом для $N \rightarrow \infty$ как размер, так и масса копии стремятся к нулю. Таким образом, с ростом $N$, с одной стороны, убывает энергия кинка, а с другой - сохраняется топологический заряд. Поэтому становится ясно, что в принципе возможен процесс сжатия ("коллапса") кинка вплоть до бесконечно малого размера и нулевой массы. Как и сами квантовые копии, такой процесс сжатия кинка является чисто непертурбативным эффектом и весьма существенно зависит от деталей динамики системы. В настоящей работе мы аналитически и численно покажем, что при отсутствии в системе дополнительных динамических законов сохранения, запрещающих испускание мезонов кинком, такой процесс сжатия действительно возможен, и качественно исследуем его динамику.

Следует подчеркнуть, однако, что, как и сами кинки, полюсные члены и тем самым наша вычитательная процедура лежат вне рамок теории возмущений. Поэтому, поскольку у нас пока нет способов суммировать весь ряд теории возмущений и тем самым провести более детальное исследование соответствия между полюсными членами и ультрафиолетовыми расходимостями теории, мы вынуждены ограничиться здесь лишь приведенными выше наводяшими соображениями для аргументации в пользу такого вычитания.

Чтобы избежать ненужных технических усложнений, мы рассмотрим $(1+1)$-мерную 
нелинейную модель для скалярного поля $\varphi(x, t)$ с лагранжианом

$$
\mathcal{L}(\varphi)=\frac{1}{2}\left(\partial_{\mu} \varphi\right)^{2}-V(\varphi)
$$

которое допускает классическое решение в виде топологического кинка или солитона

$$
\varphi_{c}(x)=u(x-X)
$$

Масштабируя переменные $[1,6]$, в $(1+1)$-мерном случае всегда можно в качестве безразмерного параметра разложения выбрать эффективную постоянную Планка $\hbar$, т.е. величину, входящую в правую часть канонических коммутационных соотношений

$$
[\varphi(x, t), \pi(y, t)]=i \hbar \delta(x-y)
$$

В этом случае классическое решение имеет нулевой порядок малости $O(1)$, амплитуда квантовых флуктуаций поля (мезонов) будет порядка $\sqrt{\hbar}$, а их энергия пропорциональна $\hbar$.

Структура работы следуюшая. В разделе 2 дается краткий обзор схемы восстановления ковариантности кинковой составляюшей с помошью групповых координат Ньютона-Вигнера. В разделе 3 мы подробно исследуем расходимости, связанные с дифференцированием конечных разрывов, и сформулируем процедуру их вычитания в рамках лоренц-ковариантного подхода к квантованию кинков из раздела 2. Раздел 4 посвяшен детальному анализу свойств квантовых копий классического кинкового решения, возникающих из разностной структуры динамических уравнений на профильную функцию и массу кинка. Матричные элементы оператора поля в кинковом секторе вычислены в разделе 5, там же обсуждается построение классического предела для квантовых копий. Возможность квантовых переходов между кинковыми решениями с разными $N$ исследована в разделе 6 , асимптотический закон такого "коллапса" обсуждается в разделе 7 , результаты численного анализа приведены в разделе 8. Заключительные замечания и обшие выводы сфформулированы в разделе 9.

\section{2. ОПЕРАТОРЫ КООР ДИНАТ НЬЮТОНА-ВИГНЕРА И КОВАРИАНТНОЕ КВАНТОВАНИЕ 1+1-МЕРНЫХ КИНКОВ}

Традиционный подход к введению коллективных координат состоит в замене параметра $X$ в уравнении (1.4) коллективной переменной $X(t)$, что восстанавливает нарушенную трансляционную инвариантность (но не лоренцеву) и позволяет строить состояния с хорошо определенным полным импульсом [7-11]. В рамках пертурбативного подхода по обратным степеням массы кинка этой замены оказывается вполне достаточно для решения таких задач, как определение спектра квантовых возбуждений, квантовой поправки к массе кинка, кинк-мезонного рассеяния и т.п. Однако тот факт, что лоренц-инвариантность не восстановлена, оставляет скорость кинка "пертурбативной", т.е. малой (нерелятивистской), и произвольная скорость может быть получена только 
частичным суммированием ряда теории возмущений. Хотя такая процедура пересуммирования возможна и дает ожидаемые релятивистские выражения как для самого классического поля $[7,9,12-14]$, так и для однопетлевых поправок [15], последовательное квантование должно быть организовано таким образом, чтобы не нарушать изначальную ковариантность теории, а сохранять ее на каждом этапе вычислений.

Ковариантное квантование кинков [16-19] опирается на фундаментальный результат Ньютона и Вигнера [4], который состоит в том, что естественным определением оператора координаты центра масс квантовой системы в релятивистском случае является выражение (в $1+1$-измерениях)

$$
q=\frac{1}{2}\left(\frac{1}{H} L+L \frac{1}{H}\right)
$$

т.е. этот оператор может быть реализован как элемент обертываюшей алгебры генераторов группы Пуанкаре $H, L$ и $P$

$$
[L, H]=i \hbar P, \quad[L, P]=i \hbar H, \quad[H, P]=0 .
$$

Динамика оператора $q$ :

$$
\dot{q}=\frac{i}{\hbar}[H, q]=\frac{P}{H},
$$

в точности описывает равномерное движение центра масс со скоростью $v=P / H$. Следует подчеркнуть, что уравнение движения для коллективной координаты $X(t)$ в отличие от (2.3) содержит полевые переменные и зависит от наложенного калибровочного условия [20]. Поэтому динамика переменной $X(t)$ оказывается существенно нетривиальной в отличие от динамики $q$.

Из физического смысла оператора $q$ ясно, что он должен входить в аргументы оператора поля исключительно в комбинации $(x-q(t))[5,16-19]$. Для наших целей это удобно осушествить посредством замены

$$
x \rightarrow \xi(x, t),
$$

где

$$
\xi(x, t)=H x-P t-L=H^{1 / 2}(x-q(t)) H^{1 / 2} .
$$

Введенная таким образом безразмерная координата $\xi(x, t)$ при преобразованиях группы Пуанкаре $x^{\mu} \rightarrow x^{\prime \mu}=\Lambda_{\nu}^{\mu} x^{\nu}+a^{\mu}$ ведет себя как ковариантный скаляр, т.е. удовлетворяет условию (1.1), если в качестве генераторов унитарных преобразований $U(\Lambda, a)$ будет использоваться алгебра (2.2), именно

$$
U^{+}(\Lambda, a) \xi(x) U(\Lambda, a)=\xi\left[\Lambda^{-1}(x-a)\right]
$$

Тем самым любая $c$-числовая функция аргумента $\xi(x, t)$ также будет автоматически преобразовываться при преобразованиях группы Пуанкаре как ковариантный скаляр 
в соответствии с условием (1.1). Поэтому мы имеем возможность записать разложение поля на кинковую и мезонную части в следующем виде:

$$
\varphi(x, t)=f(\xi(x, t))+\Phi(\xi(x, t), t)
$$

Мы специально ввели здесь новое обозначение $f(\xi)$ для "классической" составляюшей поля (она является классической в том смысле, что $f(\xi)$ есть $c$-числовая функция своего аргумента), поскольку уравнение для $f(\xi)$ в обшем случае не будет тождественно совпадать с классическим из-за операторной природы аргумента $\xi(x, t)$. Оператор мезонного поля $\Phi(\xi, t)$ в разложении $(2.7)$, рассматриваемый как функция $c$-числового аргумента, коммутирует с генератором пространственных трансляций $P$, его коммутатор с $P$ не равен нулю только из-за операторного аргумента. Коммутаторы $\Phi(\xi, t)$ с остальными генераторами группы Пуанкаре определяются из условия ковариантности всего поля $\varphi(x, t)[5,16-19]$. Далее мы сконщентрируем свое внимание на свойствах кинкового поля $f(\xi, t)$, которое ковариантно по построению. При этом оператор квадрата массы кинка, определенный соотношением $M^{2}=H^{2}-P^{2}$, коммутирует со всеми генераторами группы Пуанкаре и, следовательно, со всеми с-числовыми функциями от операторного аргумента $\xi(x, t)$, поэтому во всех последуюших выкладках он может рассматриваться как $c$-число.

Следует отметить, что хотя на первый взгляд кажется, что подстановка (2.4)-(2.7) является несколько произвольной, в действительности она с неизбежностью возникает как конечный результат упомянутой выше процедуры пересуммирования ряда теории возмушений. Эта процедура может быть проведена как при прямолинейном суммировании соответствуюших секулярных членов [5, 21], так и более опосредованно через доказательство эквивалентности подстановки (2.4)-(2.7) и канонического подхода с введением коллективной переменной $X(t)$ в рамках разложения по обратным степеням массы кинка [16-19]. Таким образом, мы в некотором смысле изначально закладываем в замене (2.4)-(2.7) конечный результат любого квазиклассического разложения и сконцентрируем свое внимание на эффектах, которые не могут быть непосредственно получены ни в каком конечном порядке такого разложения. Сказанное относится и к квазиклассическим рядам, возникаюшим при применении БРСТ-процедуры для квантования в окрестности классического кинкового решения [22-24].

\section{3. ОПЕРАТОРЫ КОНЕЧНЫХ РАЗНОСТЕЙ И ПОЛЮСНЫЕ РАСХОДИМОСТИ}

Замена (2.4) сушественно меняет уравнения, описывающие кинк, которые теперь содержат конечные разности, т.е. оказываются в некотором смысле нелокальными. Это и не удивительно, достаточно вспомнить, что группа Пуанкаре “отделена" от динамики поля посредством операторного аргумента $\xi(x, t)$. Возникающие при этом операторы конечных разностей обладают рядом специфических свойств, более того, само их корректное определение не так тривиально, как может показаться на первый взгляд, поэтому необходимо подробно обсудить их вывод. Прежде всего появление в уравнении движения конечных разностей вместо дифференциальных операторов является простым 
следствием алгебры генераторов группы Пуанкаре (точнее того факта, что $H$ и $P$ сдвигают собственные значения оператора $L$ на $\pm i \hbar)$. Действительно, подставим выражение (2.7) в исходное уравнение поля

$$
\square \varphi(x, t)+V^{\prime}(\varphi(x, t))=0
$$

и удержим только члены с "классическим" полем $f(\xi(x, t))$ порядка $O(1)$. Производные $\partial_{\mu}$ от $f(\xi(x, t))$ выражаются через коммутаторы с генераторами группы Пуанкаре:

$$
\partial_{\mu} f(\xi(x, t))=\frac{i}{\hbar}\left[P_{\mu}, f(\xi(x, t))\right]
$$

Введем теперь операторы сдвигов на световом конусе

$$
K_{ \pm}=H \pm P .
$$

После очевидных выкладок имеем

$$
K_{ \pm} \xi^{n}(x, t)=[\xi(x, t) \pm i \hbar]^{n} K_{ \pm}
$$

откуда

$$
\square_{x t} \xi^{n}=\left(\frac{i}{\hbar}\right)^{2}\left[K_{+},\left[K_{-}, \xi^{n}\right]\right]=\frac{M^{2}}{\hbar^{2}}\left[(\xi+i \hbar)^{n}+(\xi-i \hbar)^{n}-2 \xi^{n}\right],
$$

где $M^{2}=K_{+} K_{-}=H^{2}-P^{2}$ есть оператор массы, коммутируюший со всеми генераторами группы Пуанкаре и тем самым с оператором $\xi(x, t)$.

Отсюда очевидно, что для целой функции $f(\xi)$ также выполняется соотношение

$$
\square f(\xi(x, t))=\sum_{n} f_{n} \square \xi^{n}(x, t)=\frac{M^{2}}{\hbar^{2}}[f(\xi+i \hbar)+f(\xi-i \hbar)-2 f(\xi)](x, t),
$$

из которого получаем следуюшее уравнение на проффильную функцию кинка:

$$
f(\xi+i \hbar)+f(\xi-i \hbar)-2 f(\xi)+\frac{\hbar^{2}}{M^{2}} V^{\prime}(f(\xi))=0
$$

Разумеется, уравнение движения будет содержать теперь операторный аргумент, но, поскольку спектр оператора $\xi(x, t)$ непрерывен и совпадает с множеством действительных чисел (оператор $\xi(x, t)$ - унитарное преобразование оператора $L$ с помощью $\exp (i H t-i P x)$, а спектр $L$ есть как раз множество действительных чисел), то оно обязано выполняться и для $c$-числового действительного аргумента $\xi$.

Однако для наших целей необходимо расширить определение оператора конечной разности, по крайней мере, на функции со счетным числом изолированных полюсов. 
Для этого рассмотрим произвольную мероморфную функцию $f(z)$, представимую по теореме Миттаг-Леффлера в виде [25]

$$
f(z)=\sum_{n} \sum_{k=1}^{m_{n}} \frac{c_{-k}^{(n)}}{\left(z-a_{n}\right)^{k}}+\sum_{k=1}^{m} c_{k} z^{k}
$$

здесь последний член соответствует целой функции с полюсом в бесконечно удаленной точке, при этом предполагается, что полюсы $a_{n}$ не лежат на прямых $\operatorname{Im} z=0, \pm \hbar$.

Казалось бы, соотношение переброса между $K_{ \pm}$и отрицательными степенями $\left(\xi-a_{n}\right)^{-k}$ должно автоматически следовать из (3.4). Однако это оказывается неверно, поскольку необходимо преж де всего корректно определить соответствующий оператор отрицательной степени. Такое определение естественно сделать через спектральное разложение по полному набору собственных векторов оператора $\xi$, а именно

$$
f(\xi)=\int d \xi|\xi\rangle f(\xi)\langle\xi|
$$

Чтобы корректно ввести такие состояния, целесообразно использовать то обстоятельство, что в пренебрежении мезонным полем мы имеем суперотбор по массе кинка $M$, поэтому естественно перейти к быстротному представлению, в котором генератор лоренцева буста $L$ имеет вид

$$
L=i \hbar \frac{\partial}{\partial \chi}
$$

канонически сопряженная к $L$ переменная $\chi$ есть быстрота, а генераторы сдвигов принимают вид $H=M \operatorname{ch} \chi, P=M \operatorname{sh} \chi$. Собственные векторы $L$ и оператора $\xi=\xi(0,0)=-L$ тогда следуюшие:

$$
\langle\chi \mid L\rangle=(2 \pi \hbar)^{-1 / 2} \exp \left(-\frac{i}{\hbar} L \chi\right), \quad\langle\chi \mid \xi\rangle=(2 \pi \hbar)^{-1 / 2} \exp \left(\frac{i}{\hbar} \xi \chi\right) .
$$

Теперь, используя определение (3.9) и полный набор (3.11), нетрудно убедиться, что перестановочные соотношения между $K_{ \pm}$и $\left(\xi-a_{n}\right)^{-k}$ уже не будут иметь столь же простой вид, как в (3.4). Именно, имеют место следующие соотношения:

$$
K_{ \pm} \frac{1}{\xi-a}=\frac{1}{\xi \pm i \hbar-a} K_{ \pm} \pm 2 \pi i K_{ \pm} \delta(\xi-a)
$$

причем член с $\delta$-функцией появляется для $K_{+}$, если $0<\operatorname{Im} a<\hbar$, и для $K_{-}$, если $0>\operatorname{Im} a>-\hbar$. Соотношение (3.12) является операторным равенством, которое элементарно проверяется вычислением матричных элементов $\left\langle\chi|\ldots| \chi^{\prime}\right\rangle$, при этом $\delta$-функция от комплексного операторного аргумента определяется через свое спектральное разложение в соответствии с определением (3.9).

С помошью разложения Миттаг-Леффлера (3.8) для произвольной мероформной функции $f(\xi)$ тогда будем иметь

$$
K_{ \pm} f(\xi)=f(\xi \pm i \hbar) K_{ \pm} \pm 2 \pi i K_{ \pm} \sum \Gamma_{n} \delta\left(\xi-a_{n}\right)
$$


где $\Gamma_{n}$ - вычеты $f(\xi)$ в полюсах $a_{n}$, и в члене с $\delta$-функциями учитываются только полюсы, расположенные в полосах $0<\operatorname{Im} a_{n}<\hbar$ для $K_{+}$и $0>\operatorname{Im} a_{n}>-\hbar$ для $K_{-}$.

Таким образом, при наличии у $f(z)$ полюсов в полосе $|\operatorname{Im} z|<\hbar$ дифференцирование оператора $f(\xi(x, t))$ приводит, помимо регулярных разностей типа $(3.7)$, к дополнительным сингулярным полюсным членам, содержашим $\delta\left(\xi-a_{n}\right)$. Нетрудно видеть, что именно эти полюсные члены приводят к расходимости в кинетической энергии такой конфигурации, т.к. последняя квадратична по производным и тем самым будет содержать интеграл по $d \xi$ с квадратом $\delta$-функций. Чтобы еще более четко прояснить связь между полюсами в полосе $|\operatorname{Im} z|<\hbar$ и расходимостями в энергии, переформулируем теперь наш подход в терминах матричных элементов от кинкового оператора поля по методу Голдстоуна-Джекива [26]. Как и ранее, мы сконцентрируем свое внимание на свойствах собственно кинкового поля, игнорируя квантовые флуктуации. Чтобы сохранить ковариантность описания, состояние кинка будем задавать в быстротном представлении вектором $|\chi\rangle$, при этом энергия-импульс кинка есть $\varepsilon=M \operatorname{ch} \chi, p=M \operatorname{sh} \chi$.

Согласно [26] определим формфактор кинка как матричный элемент оператора поля между кинковыми состояниями

$$
\begin{aligned}
\tilde{f}\left(\chi-\chi^{\prime}\right) & =\left\langle\chi|\varphi(0,0)| \chi^{\prime}\right\rangle=\int d \xi\langle\chi \mid \xi\rangle f(\xi)\left\langle\xi \mid \chi^{\prime}\right\rangle= \\
& =(2 \pi \hbar)^{-1} \int d \xi f(\xi) \exp \left(\frac{i}{\hbar} \xi\left(\chi-\chi^{\prime}\right)\right)
\end{aligned}
$$

Тогда матричный элемент оператора поля в гейзенберговом представлении может быть записан в виде

$$
\left\langle\chi|\varphi(x, t)| \chi^{\prime}\right\rangle=\exp \left\{\frac{i}{\hbar} M\left[t\left(\operatorname{ch} \chi-\operatorname{ch} \chi^{\prime}\right)-x\left(\operatorname{sh} \chi-\operatorname{sh} \chi^{\prime}\right)\right]\right\} \widetilde{f}\left(\chi-\chi^{\prime}\right) .
$$

Полученное нами уравнение движения (3.7) является прямым следствием этого соотношения. Действительно, вычислим матричный элемент от левой и правой частей исходного уравнения движения поля (3.1) между состояниями $\langle\chi|$ и $\left|\chi^{\prime}\right\rangle$. Пользуясь (3.15), получаем

$$
\begin{aligned}
\frac{\partial^{2}}{\partial t^{2}}\left\langle\chi|\varphi(x, t)| \chi^{\prime}\right\rangle= & -\frac{M^{2}}{\hbar^{2}}\left(\operatorname{ch} \chi-\operatorname{ch} \chi^{\prime}\right)^{2}\left\langle\chi|\varphi(x, t)| \chi^{\prime}\right\rangle, \\
\frac{\partial^{2}}{\partial x^{2}}\left\langle\chi|\varphi(x, t)| \chi^{\prime}\right\rangle= & -\frac{M^{2}}{\hbar^{2}}\left(\operatorname{sh} \chi-\operatorname{sh} \chi^{\prime}\right)^{2}\left\langle\chi|\varphi(x, t)| \chi^{\prime}\right\rangle, \\
\left\langle\chi|V(\varphi(x, t))| \chi^{\prime}\right\rangle= & \exp \left\{\frac{i}{\hbar} M\left[t\left(\operatorname{ch} \chi-\operatorname{ch} \chi^{\prime}\right)-x\left(\operatorname{sh} \chi-\operatorname{sh} \chi^{\prime}\right)\right]\right\} \times \\
& \times(2 \pi \hbar)^{-1} \int d \xi V(f(\xi)) \exp \left(\frac{i}{\hbar} \xi\left(\chi-\chi^{\prime}\right)\right) .
\end{aligned}
$$

С помощью формул (3.16) рассматриваемый матричный элемент приводится к виду

$$
\frac{2 M^{2}}{\hbar^{2}}\left[\operatorname{ch}\left(\chi-\chi^{\prime}\right)-1\right]\left\langle\chi|\varphi(x, t)| \chi^{\prime}\right\rangle+\left\langle\chi\left|V^{\prime}(\varphi(x, t))\right| \chi^{\prime}\right\rangle=0
$$


откуда через обратное преобразование Фурье получим в точности уравнение (3.7).

Таким образом, при выводе уравнений движения в рамках релятивистского (матричные элементы вычисляются по состояниям с определенной быстротой, а не импульсом) формализма Голдстоуна-Джекива мы получаем такую же разностную форму уравнений, что и ранее, а $f(\xi)$ есть тем самым не что иное, как преобразование Фурье от формфактора кинка в быстротном представлении. Заметим, однако, что эквивалентность уравнений (3.7) и (3.17) имеет место только для функций $f(z)$, аналитичных в полосе $|\operatorname{Im} z|<\hbar$, т.к. в противном случае обратное фурье-преобразование от кинетического члена в (3.17) не существует из-за наличия дополнительного экспоненциального множителя $\operatorname{ch}\left(\chi-\chi^{\prime}\right)$.

Аналогичную картину мы обнаружим и при вычислении энергии кинка в рамках формализма Голдстоуна-Джекива. Вследствие явной ковариантности всех построений достаточно провести это вычисление для состояний с нулевым импульсом, аналогичные соотношения для произвольных состояний могут быть получены преобразованием Лоренца. Естественным определением массы кинка является нормированный матричный элемент

$$
M[f]=\left.\frac{\left\langle M^{\prime}, \chi^{\prime}|H(\varphi)| M, \chi\right\rangle}{\left\langle M^{\prime}, \chi^{\prime} \mid M, \chi\right\rangle}\right|_{\chi=0},
$$

в котором после всех вычислений быстрота полагается равной нулю, а оператор $H(\varphi)-$ гамильтониан поля

$$
H(\varphi)=\int d x\left[\frac{1}{2}\left(\partial_{t} \varphi\right)^{2}+\frac{1}{2}\left(\partial_{x} \varphi\right)^{2}+V(\varphi)\right]
$$

Вследствие трансляционной инвариантности интегрирование по $d x$ снимается автоматически, и поскольку $f(\xi(x, t))$ коммутирует с $M$, весь матричный элемент диагонален по $M$. В результате получаем

$$
M=\left.2 \pi \hbar \delta\left(M \operatorname{sh} \chi-M \operatorname{sh} \chi^{\prime}\right) \frac{\left\langle\chi^{\prime}|\mathcal{H}(0)| \chi\right\rangle}{\left\langle\chi^{\prime} \mid \chi\right\rangle}\right|_{\chi=0}=\left.\frac{2 \pi \hbar}{M \operatorname{ch} \chi}\langle\chi|\mathcal{H}(0)| \chi\rangle\right|_{\chi=0}
$$

где $\mathcal{H}(x)$ - пространственная плотность гамильтониана.

Таким образом, мы приходим к среднему от гамильтониана по быстротным состояниям $\left.\langle\chi|\mathcal{H}(0)| \chi\rangle\right|_{\chi=0}$, при этом основной интерес представляет “кинетический” член с производными :

$$
T=\frac{1}{2}\left[\left(\partial_{t} \varphi\right)^{2}+\left(\partial_{x} \varphi\right)^{2}\right] .
$$

Следуя [26], при вычислении $\langle\chi|T| \chi\rangle$ мы вставим в матричный элемент разложение единичного оператора $\int d \chi^{\prime}\left|\chi^{\prime}\right\rangle\left\langle\chi^{\prime}\right|$ и получим

$$
\langle\chi|T| \chi\rangle=\frac{M^{2}}{2 \hbar^{2}} \int d \chi^{\prime}\left[\left(\operatorname{ch} \chi-\operatorname{ch} \chi^{\prime}\right)^{2}+\left(\operatorname{sh} \chi-\operatorname{sh} \chi^{\prime}\right)^{2}\right]\left|\widetilde{f}\left(\chi-\chi^{\prime}\right)\right|^{2} .
$$


Если теперь положить $\chi=0$ и перейти от $\widetilde{f}(\chi)$ к $f(\xi)$, выполнив интегрирование по $d \chi^{\prime}$ (не интересуясь пока сходимостью соответствующих интегралов), то выражение (3.21) можно привести к разностному виду, аналогичному (3.7) и приведенному в [6],

$$
\left.\langle\chi|T| \chi\rangle\right|_{\chi=0}=(2 \pi \hbar)^{-1} \frac{M^{2}}{2 \hbar^{2}} \int d \xi\left\{\left[\left(\cos \hbar \partial_{\xi}-1\right) f(\xi)\right]^{2}+\left[\left(\sin \hbar \partial_{\xi}\right) f(\xi)\right]^{2}\right\}
$$

Однако в общем случае такой переход от (3.21) к (3.22) является незаконным, поскольку промежуточные выражения могут содержать расходящиеся интегралы. В частности, выражение (3.21) определено далеко не при всех $f(\xi)$, при которых существет (3.22), т.к. интеграл по $d \chi^{\prime}$ может расходиться из-за экспоненциального фактора $\left[\left(\operatorname{ch} \chi-\operatorname{ch} \chi^{\prime}\right)^{2}+\left(\operatorname{sh} \chi-\operatorname{sh} \chi^{\prime}\right)^{2}\right]$ при недостаточно быстром убывании $\tilde{f}(\chi)$. В частности, это происходит, если $\widetilde{f}(\chi)$ убывает медленнее чем $\exp (-|\chi|)$. Легко проверить, что такой случай будет иметь место при попадании полюса функции $f(z)$ в полосу, ограниченную прямыми $\operatorname{Im} z= \pm \hbar$. Именно это обстоятельство делает в обшем случае незаконной смену порядка интегрирования по $d \chi$ и $d \chi^{\prime}$ при вычислении матричного элемента $\langle\chi|T| \chi\rangle$ и тем самым является формальной причиной появления бесконечной по величине разницы между выражениями (3.21) и (3.22). Эта бесконечная разница есть не что иное, как артефакт классической сингулярности, связанной с появлением разрыва у полевой конфигурации, т.к. условие попадания полюса в полосу $|\operatorname{Im} z|<\hbar$ в классическом пределе $(\hbar \rightarrow 0)$ означает, что полюс приближается к вешественной оси, что соответствует почти разрывной функции $f(\xi)$. (Конкретный пример такого рода будет рассматриваться в следуюшем разделе.) С другой стороны, вышеприведенный анализ показывает, что с помошњю (3.13) эти расходимости могут быть выделены в производных поля в виде аддитивных полюсных членов, содержащих $\delta$-функции от комплексного аргумента. Покажем теперь, не претендуя на общность формулировок, что если квантовать поле не в стандартном $x p$-представлении (координата-импульс), а в быстротном $\xi \chi$-представлении (лоренцев буст-быстрота), то такие $\delta$-функции от комплексного аргумента вообще оказываются естественной формой представления сингулярностей квантованной теории. Для удобства массу элементарного кванта поля положим равной единище $m=1$.

В стандартном $x p$-представлении каноническая пара полевых переменных имеет вид

$$
\begin{aligned}
& \varphi(x)=\frac{1}{\sqrt{2 \pi \hbar}} \int \frac{d p}{\sqrt{2 \omega_{p}}}\left[a_{p} e^{i p x / \hbar}+\text { h.c. }\right] \\
& \pi(x)=\frac{i}{\sqrt{2 \pi \hbar}} \int \sqrt{\frac{\omega_{p}}{2}} d p\left[a_{p}^{+} e^{-i p x / \hbar}-\text { h.c. }\right]
\end{aligned}
$$

где

$$
\left[a_{p}, a_{p^{\prime}}^{+}\right]=\hbar \delta_{p p^{\prime}}, \quad\left[a_{p}, a_{p^{\prime}}\right]=0 .
$$

Перейдем теперь к быстротному представлению $p=\operatorname{sh} \chi, \omega_{p}=\operatorname{ch} \chi$ с помошюю канонического преобразования операторов рождения-уничтожения

$$
a_{p}=a_{\chi} / \sqrt{\operatorname{ch} \chi}
$$


так что

$$
\left[a_{\chi}, a_{\chi^{\prime}}^{+}\right]=\hbar \delta_{\chi \chi^{\prime}}, \quad\left[a_{\chi}, a_{\chi^{\prime}}\right]=0
$$

и построим соответствующую пару полевых переменных для $\xi \chi$-представления

$$
\begin{aligned}
& \Phi(\xi)=\frac{1}{\sqrt{2 \pi \hbar}} \int \frac{d \chi}{\sqrt{2}}\left[a_{\chi} e^{i \chi \xi / \hbar}+\text { h.c. }\right] \\
& \Pi(\xi)=\frac{i}{\sqrt{2 \pi \hbar}} \int \operatorname{ch} \chi \frac{d \chi}{\sqrt{2}}\left[a_{\chi}^{+} e^{-i \chi \xi / \hbar}-\text { h.c. }\right]
\end{aligned}
$$

Коммутатор между $\Phi(\xi)$ и П $\left(\xi^{\prime}\right)$ при этом оказывается равным

$$
\left[\Phi(\xi), \Pi\left(\xi^{\prime}\right)\right]=\frac{i}{2 \pi} \int d \chi \operatorname{ch} \chi e^{i \chi\left(\xi-\xi^{\prime}\right) / \hbar}=\frac{i \hbar}{2}\left[\delta\left(\xi-\xi^{\prime}+i \hbar\right)+\delta\left(\xi-\xi^{\prime}-i \hbar\right)\right]
$$

Таким образом, каноническое квантование в $\xi \chi$-представлении уже с самого начала содержит $\delta$-функции от комплексного аргумента, вполне аналогичные тому, что наблюдается в (3.13). С другой стороны, поскольку в конечном счете именно сингулярность в каноническом коммутаторе лежит в основе всех остальных расходимостей теории, то должно быть ясно, что в быстротном представлении к аналогичному виду могут быть приведены и другие расходимости теории. Рассмотрим это на примере энергии нулевых колебаний поля, используя следующую цепочку выкладок:

$$
\Delta E=\frac{1}{2} \int \omega_{p} d p\left(a_{p} a_{p}^{+}-a_{p}^{+} a_{p}\right)=\frac{1}{2} \int \operatorname{ch} \chi d \chi\left(a_{\chi} a_{\chi}^{+}-a_{\chi}^{+} a_{\chi}\right),
$$

после чего выражаем $a_{\chi}$ через $\Phi(\xi)$ и $\Pi(\xi)$ и сводим $\Delta E$ к коммутатору между $\Phi(\xi)$ и $\Pi\left(\xi^{\prime}\right)$ :

$$
\begin{aligned}
\Delta E & =\frac{1}{2 i} \int d \xi d \xi^{\prime} \delta\left(\xi-\xi^{\prime}\right)\left[\Phi(\xi), \Pi\left(\xi^{\prime}\right)\right]= \\
& =\frac{\hbar}{2} \int d \xi d \xi^{\prime} \delta\left(\xi-\xi^{\prime}\right) \frac{1}{2}\left[\delta\left(\xi-\xi^{\prime}+i \hbar\right)+\delta\left(\xi-\xi^{\prime}-i \hbar\right)\right]
\end{aligned}
$$

Как (3.29), так и (3.30) приводят к одной и той же расходимости $\Delta E=(\hbar / 2) \int \operatorname{ch} \chi d \chi$. Сушественно, однако, что, как это следует из (3.30), естественной формой записи этой расходимости в быстротном представлении снова оказьвается представление через $\delta$-функции от комплексного аргумента.

Нам остается сделать лишш последний шаг, а именно постулировать вычитание таких $\delta$-функций от комплексного аргумента в тех случаях, когда установлена их прямая связь с расходимостями теории, которые должны устраняться из интуитивных физических соображений. Сразу оговоримся, что это вычитание означает несколько иное действие по сравнению с тем, что вкладывается в этот термин при ультрафиолетовой перенормировке в диаграммной теории возмущений. В последнем случае вычитание имеет буквальный смысл как добавление к затравочному лагранжиану теории набора контрчленов, 
содержащих расходящиеся величины, которые компенсируют ультрафиолетовые расходимости затравочной теории. При этом контрчленам удается придать достаточно наглядный смысл как бесконечной перенормировки масс и констант связи. В настоящем случае мы не умеем формулировать нашу вычитательную процедуру на таком уровне и поэтому наша процедура вычитания расходимостей сводится к постулативному вычеркиванию из промежуточных вычислений тех $\delta$-функций от комплексного аргумента, которые ответственны за данную расходимость. В частности, устранение расходимостей, связанных с дифференцированием конечных разрывов кинкового профиля, сводится к вычеркиванию членов с $\delta$-функциями в (3.13). Если принять точку зрения, что такое вычеркивание допустимо, то динамическим уравнением, определяющим профильную функцию кинка $f(\xi)$ при единственном условии мероморфности и регулярности на прямых $\operatorname{Im} z=0, \pm \hbar$ в комплексной плоскости, будет конечно-разностное уравнение (3.7). Далее нетрудно показать, что при вычислении массы кинка производные в гамильтониане поля также превратятся в операторы конечных разностей такого же вида. По существу, мы это уже показали при вычислении матричного элемента от кинетической энергии кинка в виде (3.22) в рамках метода Голдстоуна-Джекива. Для полноты картины приведем теперь чисто операторный вывод массы кинка как функционала от $f(\xi)$ в рамках ковариантной подстановки (2.4). Переписывая частные производные в терминах коммутаторов с $K_{ \pm}$и снова опуская члены с $\delta$-функциями, ответственные за появление расходимости в энергии, получаем

$$
\begin{aligned}
T= & \frac{1}{2}\left[\left(\partial_{t} \varphi\right)^{2}+\left(\partial_{x} \varphi\right)^{2}\right]= \\
= & \frac{i^{2}}{4 \hbar^{2}} \sum_{ \pm}\left\{K_{ \pm}[f(\xi)-f(\xi \mp i \hbar)][f(\xi \pm i \hbar)-f(\xi)] K_{ \pm}\right\}= \\
= & \frac{i^{2}}{2 \hbar^{2}}\{H[f(\xi)-f(\xi-i \hbar)][f(\xi+i \hbar)-f(\xi)] H+ \\
& \quad+P[f(\xi)-f(\xi-i \hbar)][f(\xi+i \hbar)-f(\xi)] P\}
\end{aligned}
$$

при прежних условиях на функцию $f(\xi)$ (мероморфность и регулярность на прямых $\operatorname{Im} z=0, \pm \hbar)$. Массу кинка снова определяем как матричный элемент (3.18), а для вычисления $\langle\chi|\mathcal{H}(0)| \chi\rangle$ вставим разложение единицы $\int d \xi|\xi\rangle\langle\xi|$. После ряда очевидных преобразований окончательное уравнение для массы принимает вид

$$
M^{2}=\frac{M^{2}}{2 \hbar^{2}} \int d \xi[f(\xi+i \hbar)-f(\xi)][f(\xi-i \hbar)-f(\xi)]+\int d \xi V(f(\xi)),
$$

при этом кинетический член в (3.32) в точности воспроизводит выражение (3.22) для матричного элемента $\langle\chi|T| \chi\rangle$, полученное с помошью метода Голдстоуна-Джекива.

Используя эти результаты, нетрудно проверить, что при таком вычитании расходимостей мы не должны добавлять никаких конечных величин, т.е. конечный произвол в нашей процедуре вычитания отсутствует. Для этого заметим, что разложение (3.7) по степеням $\hbar$ с точностью до $O\left(\hbar^{2}\right)$ воспроизводит классическое уравнение на кинковую составляющую

$$
-M_{0}^{2} f^{\prime \prime}(\xi)+V^{\prime}(f)=0
$$


где $M_{0}$ - классическая масса кинка. Поэтому если "квазиклассический” предел $\hbar \rightarrow 0$ для $f(\xi)$ существует, то он есть просто

$$
f(\xi)=\varphi_{c}\left(\xi / M_{0}\right),
$$

где $\varphi_{c}(x)$ - классическое решение (1.2). Далее, полагая $\hbar \rightarrow 0$ в соотношении (3.32), с той же точностью $O\left(\hbar^{2}\right)$ получаем

$$
M_{0}^{2}=\int d \xi\left[\frac{M_{0}^{2}}{2} f^{\prime}(\xi)^{2}+V(f(\xi))\right],
$$

откуда с учетом соотношения (3.34) находим, что в "квазиклассическом" пределе масса кинка определяется выражением

$$
M_{0}=\int d x\left[\frac{1}{2} \varphi_{c}^{\prime}(x)^{2}+V\left(\varphi_{c}(x)\right)\right],
$$

что есть не что иное, как его классическая энергия.

Формулы (3.33)-(3.36) полезны в двух отношениях. Прежде всего само наличие такого предельного перехода к классической теории обусловлено тем, что классическое решение по определению является гладким в том смысле, что не может иметь полюсов в полосе $|\operatorname{Im} z|<\hbar$. Для таких гладких полей наш подход не означает ничего принципиально нового по сравнению с традиционными схемами квантования, т.к. полюсных членов не будет как таковых. Именно поэтому квазиклассическое квантование в окрестности классического решения на основе подстановки (2.4)-(2.7) оказывается эквивалентным каноническому квантованию кинка в терминах коллективных координат, что и было продемонстрировано в [16-19]. С другой стороны, естественно потребовать, чтобы наша вычитательная процедура никак не сказывалась на свойствах гладких конфигураций типа (3.34). Таким образом, мы фиксируем конечный произвол в нашей схеме по крайней мере до $O\left(\hbar^{2}\right)$, что вполне достаточно для наших целей.

Таким образом, изложенная выше процедура вычитания расходяшихся величин в рамках лоренц-ковариантного квантования кинка с помошью операторов координат Ньютона-Вигнера однозначным образом приводит к конечно-разностной структуре динамических уравнений на массу и профильную функцию кинка в виде (3.7) и (3.32). Следует также отметить, что мы использовали естественную для лоренц-ковариантного подхода релятивистскую систему единищ, в которой скорость света полагается равной единище $c=1$. Если восстановить скорость света, коммутационные соотношения $(2.2)$ перепишутся в виде

$$
[L, P]=i \hbar \frac{H}{c^{2}},
$$

вследствие чего шаг в операторах конечных разностей окажется пропорциональным $\hbar / c$. Поэтому в нашем подходе на самом деле имеются два независимых параметра, первый из которых

$$
\hbar^{\prime}=\hbar / c
$$


и входит в конечные разности, тем самым описывая существенно релятивистские квантовые эффекты, а другой $-\sqrt{\hbar}-$ параметр разложения для учета квантовых флуктуаций в окрестности кинка.

\section{4. КВАНТОВЫЕ КОПИИ}

Основным следствием появления операторов конечных разностей в динамических уравнениях теории является возникновение специфических решений, которые мы будем назьвать "квантовыми копиями” классических кинков. Смысл этого утверждения состоит в том, что у конечно-разностного уравнения (3.7) в отличие от классического уравнения (3.33) сушествуют и другие решения, которые в некотором смысле "генерируются" классическим, но не имеют регулярного поведения при $\hbar \rightarrow 0$. Построение таких решений для одномерных полевых моделей подробно обсуждено в [6]. Оказывается, что если использовать разложение в ряды Дирихле, то можно построить семейство решений (3.7), которые нумеруются целочисленным индексом $N$, при этом

$$
f_{N}(\xi)=\varphi_{c}\left(\alpha_{N} \xi\right)
$$

где

$$
\alpha_{N}=\frac{1}{M_{N}}+\frac{2 \pi N}{m \hbar}
$$

здесь $M_{N}=M\left[f_{N}\right]$ - масса соответствуюшего решения, а $m$ - масса элементарного кванта поля (мезона).

При $N=0$ мы воспроизводим уже известный ответ (3.34) для "квазиклассического" предела теории, и это единственный случай, когда решение регулярно при $\hbar \rightarrow 0$. При $N \neq 0$ массы квантовых копий $M_{N}$ определяются из (3.32), что при малых $\hbar$ дает

$$
M_{N}=\sqrt{\frac{M_{0}}{\left|\alpha_{N}\right|}} \simeq \sqrt{\frac{\hbar}{|N|}}\left(\frac{M_{0} m}{2 \pi}\right)^{1 / 2} .
$$

Тем самым массы квантовых копий пропорциональны $\sqrt{\hbar}$ и занимают промежуточное положение между массой классического кинка, которая есть $O(1)$, и характерной энергией вторично квантованных возбуждений поля $\hbar \omega_{k}$. Более того, из $(4.3)$ видно, что $M_{N}$ убывает с ростом $|N|$. Необходимо подчеркнуть, что в действительности все решения вида (4.1) (включая и случай $N=0$ ) являются приближенными, т.к. уравнение (3.7) при подстановке в него функций (4.1) имеет невязку $O(\hbar)$ [6], т.е. порядка однопетлевых поправок. Однако поскольку в настоящей работе мы рассматриваем только свойства собственно кинковых конфигураций, которые определяются главными членами соответствующих разложений, то такими поправками старших порядков мы будем пренебрегать.

Теперь рассмотрим все эти эффекты более подробно на примере стандартной $\varphi^{4}$-модели. Потенциал самодействия в этом случае имеет вид

$$
V(\varphi)=\frac{1}{2}\left(1-\varphi^{2}\right)^{2}
$$


что соответствует кинковому решению

$$
\varphi_{c}(x)=\operatorname{th} x
$$

Квантовые копии такого кинка будут иметь профили

$$
f_{N}(\xi)=\operatorname{th} \alpha_{N} \xi
$$

где $\alpha_{N}=1 / M_{N}+\pi N / \hbar$, поскольку масса мезона в данном случае $m=2$.

Функции $f_{N}(\xi)$ являются мероморфными с полюсами первого порядка в точках $a_{n}=i \pi(n+1 / 2) / \alpha_{N}$. Для малых $\hbar$ прямая $\operatorname{Im} z=\hbar$ проходит между $(N-1)$-м и $N$-м полюсами, причем расстояния до них практически одинаковы. Следовательно, для $N$-го решения внутрь полосы, ограниченной прямыми $\operatorname{Im} z= \pm \hbar$, попадают $2 N$ полюсов, поэтому аналитическое продолжение на прямые $\operatorname{Im} z= \pm \hbar$ с помошью степенного разложения невозможно. Величина $f_{N}(\xi \pm i \hbar)$ должна быть определена через разложение th $z$ в ряд простых дробей (что и есть в данном случае разложение Миттаг-Леффлера)

$$
\text { th } z=\sum_{n=0}^{\infty} \frac{2 z}{z^{2}+\pi^{2}\left(n+\frac{1}{2}\right)^{2}}
$$

либо путем представления $\operatorname{sh} z$ и $\operatorname{ch} z$ в виде бесконечных произведений.

Как уже было указано, конфигурации (4.6) не точные решения уравнения (3.7). Однако легко найти “точное уравнение”, для которого они являются решениями:

$$
\square f_{N}+2 \frac{\operatorname{tg}^{2} \beta}{\beta^{2}} \frac{f_{N}\left(f_{N}^{2}-1\right)}{1+f_{N}^{2} \operatorname{tg}^{2} \beta}=0,
$$

где $\beta=\hbar / M_{N}$. При малых $\hbar$ параметр $\beta$ имеет порядок малости $O(\sqrt{\hbar})$. С учетом того, что $\left|f_{N}(\xi)\right| \leq 1$ при любых $\xi$, разница между потенциальным членом в (4.8) и "правильным" потенщиальным членом $2 f_{N}\left(f_{N}^{2}-1\right)$ имеет порядок малости $O(\hbar)$ равномерно по всей оси, что и утверждалось выше.

Проиллюстрируем теперь основные свойства квантовых копий на примере кинков (4.6). Их топологический заряд $Q_{N}$ совпадает с зарядом "исходного" классического кинка $Q= \pm 1$. При $\hbar \rightarrow 0$ либо при $|N| \rightarrow \infty$ их форма стремится к разрывному решению

$$
f_{N}(\xi) \rightarrow f_{\infty}(\xi)=\operatorname{sign} \xi,
$$

которое является решением классического (обычного, содержашего операторы дифференширования) уравнения движения (3.33) при всех $\xi$, кроме $\xi=0$. Из (4.3) следует, что масса предельной копии $f_{\infty}(\xi)$ тождественно равна нулю, тогда как в классической теории поля выражение для энергии такого решения содержало бы слагаемое $\int d x \delta^{2}(x)$, т.е. энергия была бы бесконечной. Этот результат является прямым следствием нашей 
вычитательной процедуры с вычеркиванием $\delta$-функций и фиксированием конечного произвола по квазиклассическому пределу для гладких конфигураций поля, которая приводит к операторам конечных разностей в кинетическом члене в гамильтониане поля и в конечном счете к нулевой массе разрывного решения.

Для конфигураций (4.6) этот эффект можно сделать еще более наглядным, заметив, что при малых $\hbar$ или больших $|N|$ они могут быть приближенно записаны в виде

$$
f_{N}(\xi) \simeq \operatorname{th}\left(\frac{\pi N}{\hbar} \xi\right)
$$

и являются тем самым $i \hbar$-периодическими функциями, удовлетворяющими соотношению $f(\xi \pm i \hbar)=f(\xi)$. Именно поэтому выражение для "кинетического члена" в гамильтониане тож дественно обратится в нуль, а "потенциальный" же член в энергии отличен от нуля только в переходной области, где $|f(\xi)| \neq 1$, т.е. стремится к нулю, когда размер этой области стремится к нулю при $\hbar \rightarrow 0$ либо $|N| \rightarrow \infty$.

В отношении копий с большими $|N|$ необходимо также заметить следуюшее. На самом деле для $|N|$ есть ограничение сверху $|N| \leq 1 / \hbar$, т.к. в рамках нашего подхода для копий существует очевидная нижняя грань по энергии порядка $O(\hbar)$, которая обусловлена следуюшими основными причинами. Во-первых, при стремлении энергии к нулю оказывается плохо определенным сам оператор координаты центра масс поля (2.1), который лежит в основе лоренц-ковариантного описания кинкового сектора с помощью подстановки (2.4). Во-вторых, мы трактуем возникновение кинка (или его квантовой копии) как главный эффект взаимодействия в системе и на этом основании пренебрегаем квантовыми флуктуациями поля, что верно лишь, пока энергия кинка сушественно больше характерной энергии мезонов $\hbar \omega_{k}$, иначе все рассмотрение надо проводить с учетом мезонной компоненты на равных основаниях с кинковой составляющей, что может до неузнаваемости исказить всю картину. Поэтому вопрос о статусе копий с $|N| \geq 1 / \hbar$ и связанных с ними эффектах лежит вне рамок нашего рассмотрения, и далее под предельной конфигурацией $f_{\infty}(\xi)$ мы будем понимать квантовую копию с максимальным допустимым в нашем подходе $|N| \simeq 1 / \hbar$ и минимальной допустимой энергией порядка $O(\hbar)$, при которой еше можно рассматривать квантовые флуктуации как поправки в рамках стандартного квазиклассического разложения по $\sqrt{\hbar}$ (если это вообще возможно).

\section{5. МАТРИЧНЫЕ ЭЛЕМЕНТЫ ОПЕРАТОРА ПОЛЯ ДЛЯ КВАНТОВЫХ КОПИЙ}

Рассмотрим теперь принципиальный для самосогласованности всего подхода вопрос о сушествовании классического предела для конфигураций типа квантовых копий. Для этого, следуя работе [16], вычислим матричный элемент поля в состоянии, которое в квазиклассическом пределе может быть отождествлено с состоянием материальной точки в классической механике, т.е. в состоянии, описываемом гауссовым пакетом $\Psi$, локализованным в данной точке и с дисперсией, имеющей “квантовый” порядок малости. В данном случае аналогами квантово-механических координаты и импульса служат 
переменные $L$ и $\chi$, соответственно. Итак, рассмотрим

$$
\begin{aligned}
|\Psi\rangle & =\int d \chi \Psi(\chi)|\chi\rangle \\
\Psi(\chi) & =(\pi \hbar \eta)^{-1 / 4} \exp \left[-\frac{i}{\hbar} L_{0} \chi-\frac{\left(\chi-\chi_{0}\right)^{2}}{2 \hbar \eta}\right]
\end{aligned}
$$

где средние значения $\chi$ и $L$ равны $\chi_{0}$ и $L_{0}$, а их дисперсии $-\hbar \eta / 2$ и $\hbar / 2 \eta$, соответственно.

Чтобы вычислить матричный элемент $\langle\Psi|\varphi(x, t)| \Psi\rangle$, представим $\varphi(x, t)$ в виде

$$
\begin{aligned}
\varphi(x, t) & =\exp \left(\frac{i}{h} P^{\mu} x_{\mu}\right) \varphi(0,0) \exp \left(-\frac{i}{h} P^{\mu} x_{\mu}\right)= \\
& =\int d \lambda \tilde{f}(\lambda) e^{i \lambda \xi} \exp \left[\frac{i}{h}\left(P^{\mu}(\lambda)-P^{\mu}\right) x_{\mu}\right]
\end{aligned}
$$

где

$$
P^{\mu}(\lambda)=e^{-i \lambda \xi} P^{\mu} e^{i \lambda \xi}=\left[\Lambda^{-1}(\hbar \lambda)\right]_{\nu}^{\mu} P^{\nu}
$$

и $\Lambda_{\nu}^{\mu}(\chi)$ - матрица лоренцева буста

$$
\Lambda_{\nu}^{\mu}(\chi)=\left(\begin{array}{cc}
\operatorname{ch} \chi & \operatorname{sh} \chi \\
\operatorname{sh} \chi & \operatorname{ch} \chi
\end{array}\right)
$$

В отличие от кинкового формфактора Голдстоуна-Джекива (3.14) фурье-образ $\widetilde{f}(\lambda)$, введенный в (5.2), не содержит постоянной Планка:

$$
\tilde{f}(\lambda)=\frac{1}{2 \pi} \int d \xi e^{-i \lambda \xi} f(\xi)
$$

Поэтому для любого $N$ получаем

$$
\widetilde{f}_{N}(\lambda)=\frac{1}{2 \pi} \int d \xi e^{-i \lambda \xi} \varphi_{c}\left(\alpha_{N} \xi\right)=\frac{1}{\left|\alpha_{N}\right|} \widetilde{\varphi}_{c}\left(\frac{\lambda}{\alpha_{N}}\right)
$$

где $\tilde{\varphi}_{c}(\lambda)$ - преобразование Фурье функции $\varphi_{c}(\xi)$ вида $(5.5)$ и, следовательно, также не содержит постоянной Планка $\hbar$.

Подставляя (5.2) в матричный элемент поля, получим

$$
\begin{aligned}
\langle\Psi|\varphi(x, t)| \Psi\rangle & =\frac{1}{\hbar\left|\alpha_{N}\right|} \int d \chi d \chi^{\prime} \Psi^{*}\left(\chi^{\prime}\right) \widetilde{\varphi}_{c}\left(\frac{\chi-\chi^{\prime}}{\hbar \alpha_{N}}\right) \Psi(\chi) \times \\
& \times \exp \left\{\frac{i}{h}\left[M_{N}\left(\left(t \operatorname{ch} \chi^{\prime}-x \operatorname{sh} \chi^{\prime}\right)-(t \operatorname{ch} \chi-x \operatorname{sh} \chi)\right)-L_{0}\left(\chi-\chi^{\prime}\right)\right]\right\},
\end{aligned}
$$

где $M_{N}$ - масса квантовой копии с индексом $N$.

В представлении, где быстрота играет роль импульса, все пространство естественным образом разбивается на квадранты - пространственно- и времениподобные. Мы 
проведем выкладки для пространственноподобных квадрантов $t=R \operatorname{sh} \zeta, x=R \operatorname{ch} \zeta$, для времениподобных они могут быть проведены совершенно аналогичным образом (кроме того, соответствуюший результат может быть получен просто посредством аналитического продолжения).

Учитывая сказанное, соотношение (5.7) можно представить в виде

$$
\begin{aligned}
\langle\Psi|\varphi(x, t)| \Psi\rangle= & \frac{2}{\left|\alpha_{N}\right| \sqrt{\pi \hbar \eta}} \int d y d z \exp \left(-\frac{y^{2}+z^{2}}{\hbar \eta}\right) \widetilde{\varphi}_{c}\left(\frac{2 y}{\hbar \alpha_{N}}\right) \times \\
& \times \exp \left\{\frac{2 i}{\hbar}\left[M_{N} R \operatorname{sh} y \operatorname{ch}\left(z-z_{0}\right)-L_{0} y\right]\right\}
\end{aligned}
$$

где $z_{0}=\zeta-\chi_{0}$.

Интегрирование в правой части (5.8) благодаря гауссову обрезающему фактору эффективно идет по области малых $y$, именно по окрестности $|y|<\sqrt{\hbar}$. В этой области можно заменить sh $y$ на $y$ и провести интегрирование по $d y$ :

$$
\begin{aligned}
\langle\Psi|\varphi(x, t)| \Psi\rangle= & \frac{(\pi \hbar)^{-3 / 2}}{\left|\alpha_{N}\right| \sqrt{\eta}} \int d z d \xi \exp \left(-\frac{z^{2}}{\hbar \eta}\right) \varphi_{c}(\xi) \times \\
& \times \int d y \exp \left\{-\frac{y^{2}}{\hbar \eta}+\frac{2 i y}{\hbar}\left[M_{N} R \operatorname{ch}\left(z-z_{0}\right)-L_{0}-\frac{\xi}{\alpha_{N}}\right]\right\}= \\
= & \frac{1}{\pi \hbar\left|\alpha_{N}\right|} \int d z \exp \left(-\frac{z^{2}}{\hbar \eta}\right) \int d \xi \varphi_{c}(\xi) \times \\
& \times \exp \left\{-\eta \frac{\left[M_{N} R \operatorname{ch}\left(z-z_{0}\right)-L_{0}-\xi / \alpha_{N}\right]^{2}}{\hbar}\right\} .
\end{aligned}
$$

На этом этапе вычислений мы должны рассмотреть отдельно два случая - "классический" кинк и его квантовые копии. Это связано с тем, что при $N \neq 0$ величина $\hbar \alpha_{N}$ практически не зависит от $\hbar$, а при $N=0$ имеем $\alpha_{0}=1 / M_{0}$ и поэтому $\hbar \alpha_{N}$ оказывается пропорциональной $\hbar$.

В первом случае - при $N=0$ и малых $\hbar$ интеграл по $d \xi$ может быть оценен посредством стандартного метода перевала:

$$
\int d \xi \rightarrow\left|\alpha_{0}\right| \sqrt{\pi \hbar / \eta} \varphi_{c}\left[\alpha_{0}\left(M_{0} R \operatorname{ch}\left(z-z_{0}\right)-L_{0}\right)\right]
$$

Оставшийся интеграл по $d z$ может быть вычислен совершенно аналогичным образом, и окончательный ответ для матричного элемента поля принимает вид

$$
\begin{aligned}
\left.\langle\Psi|\varphi(x, t)| \Psi\rangle\right|_{N=0} & \rightarrow u\left[\alpha_{0}\left(M_{0} R \operatorname{ch} z_{0}-L_{0}\right)\right]= \\
& =u\left(\frac{H x-P t-L_{0}}{M_{0}}\right)=u\left(\frac{x-v t-x_{0}}{\sqrt{1-v^{2}}}\right) .
\end{aligned}
$$

Здесь $v=$ th $\chi_{0}$ и $x_{0}=L_{0} /\left(M_{0} \operatorname{ch} \chi_{0}\right)$, т.е., как и следовало ожидать, для регулярного решения с $N=0$ матричный элемент поля в пределе $\hbar \rightarrow 0$ совпадает с исходным 
классическим кинком. Отметим также, что явная ковариантность процедуры квантования позволяет получить из матричных элементов поля классический кинк с произвольными координатой и скоростью.

Во втором случае - при $N \neq 0$ и малых $\hbar$ интеграл по $d \xi$ требует более детального анализа, поскольку $\hbar \alpha_{N}$ теперь не мало. Заменяя $\alpha_{N}$ на $2 \pi N / \hbar m$, мы можем переписать (5.9) в виде

$$
\begin{aligned}
\left.\langle\Psi|\varphi(x, t)| \Psi\rangle\right|_{N \neq 0}= & \frac{m}{2 \pi^{2} N} \int d z d \xi \exp \left(-\frac{z^{2}}{\hbar \eta}\right) \varphi_{c}(\xi) \times \\
& \times \exp \left\{-\eta \frac{\left[M_{N} R \operatorname{ch}\left(z-z_{0}\right)-L_{0}\right]^{2}}{\hbar}+\right. \\
& \left.\quad+\frac{m \eta \xi}{\pi N}\left[M_{N} R \operatorname{ch}\left(z-z_{0}\right)-L_{0}\right]-\frac{\hbar \eta m^{2}}{(2 \pi N)^{2}} \xi^{2}\right\} .
\end{aligned}
$$

Для малых $\hbar$ можно представить интеграл по $d \xi$ следуюшим образом:

$$
\int d \xi \varphi_{c}(\xi) e^{\alpha \xi-\beta \xi^{2}}=\frac{1}{\sqrt{\beta}} \int d s \varphi_{c}\left(\frac{s}{\sqrt{\beta}}\right) e^{\gamma s-s^{2}}
$$

где

$$
\gamma=\frac{\alpha}{\sqrt{\beta}}=2 \sqrt{\frac{\eta}{\hbar}}\left[M_{N} R \operatorname{ch}\left(z-z_{0}\right)-L_{0}\right] .
$$

При этом $\gamma$ в действительности не зависит от $\hbar$, поскольку для квантовых копий $M_{N}=O(\sqrt{\hbar})$. Учитывая, что $\beta \rightarrow 0$ при $\hbar \rightarrow 0$, мы можем заменить $\varphi_{c}(s / \sqrt{\beta})$ всюду в эффективной области интегрирования $|s|<1$ на $\varphi_{c}( \pm \infty)$. В результате, если ограничиться типичным случаем нечетного топологического кинка, в первом приближении мы можем положить

$$
\varphi_{c}\left(\frac{s}{\sqrt{\beta}}\right) \rightarrow \frac{Q}{2} \operatorname{sign} s,
$$

откуда

$$
\int d \xi \rightarrow \frac{2 \pi N Q}{m \sqrt{\hbar \eta}} \exp \left\{\eta \frac{\left[M_{N} R \operatorname{ch}\left(z-z_{0}\right)-L_{0}\right]^{2}}{\hbar}\right\} \int_{0}^{\gamma / 2} d s e^{-s^{2}}
$$

Подставляя это выражение в (5.12), возьмем интеграл стандартным методом перевала (поскольку $\gamma$ не зависит от $\hbar$ ). Окончательно для матричного элемента поля от квантовой копии с индексом $N$ в классическом пределе $(\hbar \rightarrow 0)$ получаем

$$
\langle\Psi|\varphi(x, t)| \Psi\rangle_{N \neq 0} \rightarrow \frac{Q}{2} \operatorname{erf}\left(\frac{H x-P t-L_{0}}{\sqrt{\hbar / \eta}}\right),
$$

где $\operatorname{erf}(z)$ - интеграл ошибок. Следует подчеркнуть, что erf появился исключительно по той причине, что в качестве аппроксимации квазиклассического состояния материальной точки мы выбрали гауссов пакет. При другой форме пакета ответ, разумеется, изменится. При этом профильная функция копии никак не участвует в формировании 
ответа для матричного элемента (по ходу выкладок мы провели замену $\varphi_{c}(s / \sqrt{\beta})$ на $\varphi_{c}( \pm \infty)$, т.е. использовали только топологические свойства квантовой копии, совпадаюшие с топологией классического кинка).

Как и в случае $N=0$, среднее (5.17) может быть представлено в виде движушегося кинка с профилем интеграла ошибок

$$
\langle\Psi|\varphi(x, t)| \Psi\rangle_{N \neq 0} \rightarrow \frac{Q}{2} \operatorname{erf}\left(\frac{1}{d_{N}} \frac{x-v t-x_{N}}{\sqrt{1-v^{2}}}\right)
$$

где $v=$ th $\chi_{0}, x_{N}=L_{0} /\left(M_{N} \operatorname{ch} \chi_{0}\right)$ и

$$
d_{N}=\sqrt{\hbar / \eta} / M_{N} \simeq \sqrt{N / \eta}\left(2 \pi / M_{0} m\right)^{1 / 2} .
$$

Кинк (5.18) воспроизводит топологические свойства исходного классического решения, но имеет другую профильную функцию. Следует подчеркнуть, что ответ (5.18) справедлив при вполне определенных предположениях. Прежде всего мы полагали $\hbar \rightarrow 0$. Кроме того, мы неявно предполагали, что величина $\eta$ не слишком отличается от единицы. Собственно именно этим обстоятельством и объясняется "выпадение" формы исходного решения из квазиклассического среднего для копий. Локализация кинка происходит с точностью $O(\sqrt{\hbar / \eta})$ (это размер гауссова пакета), в то время как размер переходной области в профильной функции при $N \neq 0$ имеет порядок $O(\hbar)$, т.е. точная форма кинка не наблюдаема. В результате окончательный ответ соответствует переходной области размера $\sqrt{N / \eta}$ и не стремится к нулю при $\hbar \rightarrow 0$ или $N \rightarrow \infty$. Разумеется, при возрастании $\eta$ размер исходного пакета и размер переходной области в (5.18) убывают, а дисперсия $\chi$ и, следовательно, неопределенность в импульсе растут.

Возникает естественный вопрос, в какой степени мы можем воспроизвести если не форму исходной полевой конфигурации $f_{N}(\xi)$, то хотя бы ее размер? С одной стороны, очевидно, что, полагая $\eta$ произвольно малым (скажем, $\left.O\left(\hbar^{2}\right)\right)$, мы, как и в случае классического кинка с $N=0$, в точности воспроизведем форму $f_{N}(\xi)$. Однако полученный результат нельзя будет рассматривать как разумный классический предел теории: параметры соответствуюшего волнового пакета (время распльвания и кинетическая энергия, связанная с неопределенностью импульса) будут совершенно не квазиклассическими. Таким образом, мы должны дополнительно потребовать, чтобы дисперсия импульса была ограничена "квантовым" значением $O(\hbar)$. Существенной особенностью квантовых копий является то, что их масса имеет порядок малости $O(\sqrt{\hbar})$, поэтому рассматриваемая "квантовая механика материальной точки” не является вполне стандартной. В частности, полагая, что кинк как целое не движется $\left(\chi_{0}=0\right)$, для средней кинетической энергии получаем

$$
\frac{1}{2} M_{N}\left\langle\Psi\left|\chi^{2}\right| \Psi\right\rangle=O\left(\hbar^{3 / 2} \eta\right)
$$

Аналогично скорость распльвания пакета пропорциональна $\hbar /\left(M_{N} \eta\right)=O(\sqrt{\hbar} / \eta)$. Отсюда видно, что для достижения правильных квазиклассических характеристик пакета нам следует ограничить $\eta$ условием $\eta \simeq O\left(\hbar^{-1 / 2}\right)$. При этом кинетическая энергия 
и скорость распльвания пакета будут иметь порядок $O(\hbar)$, как и должно быть в "настоящем" квазиклассическом случае, а размер переходной области в среднем (5.18) будет пропорционален $O\left(\hbar^{1 / 4}\right)$, что и составляет наилучшее возможное приближение к исходному размеру решения $f_{N}(\xi)$, которьй пропорционален $\hbar$. При этом размер кинка (5.18) стремится к нулю при $\hbar \rightarrow 0$, и поэтому разумный классический предел теории для квантовых копий сушествует и действительно соответствует разрывному решению с нулевой массой.

\section{6. СОлИТОНнЫЙ коллАПС В ОДНОМОДОВОМ ПРИБЛИЖЕНИИ}

Из энергетических соображений очевидно, что ввиду убывания энергии копии с ростом $|N|$ каждое из кинковых состояний с конечным $|N|$ будет неустойчиво относительно туннелирования в направлении больших $|N|$, а возникающий избыток энергии будет при этом переходить в возбуждение когерентных мезонных волн. Этот процесс может быть назван процессом "сжатия" или "коллапса" кинка, т.к. при этом топологический заряд конфигурации $Q$ не меняется.

Чтобы описать процесс такого коллапса, необходимо ввести новую динамическую переменную, эволюция которой описывала бы трансформацию полевой конфигурации $f_{N 1}(\xi)=\varphi_{c}\left(\alpha_{N 1} \xi\right)$ в конфигурацию $f_{N 2}(\xi)=\varphi_{c}\left(\alpha_{N 2} \xi\right)$. Наиболее простой способ ввести такую переменную - это записать полевую конфигурацию в виде

$$
f(\xi, t)=c(t) f_{N 2}(\xi)+(1-c(t)) f_{N 1}(\xi) .
$$

Коэффициенты $c_{N_{1}}=c(t)$ и $c_{N_{2}}=1-c(t)$ при $f_{N 1}$ и $f_{N 2}$ выбираются таким образом, чтобы вдали от кинка (от переходной области) поле вовсе не зависело от $c(t)$, все изменения происходят только в окрестности кинка. Это гарантирует конечность энергии всех промежуточных конфигураций при любом конечном $c(t)$. Очевидно, что переход $f_{N 1} \rightarrow f_{N 2}$ описывается некоторой траекторией $c\left(t_{1}\right)=0 \rightarrow c\left(t_{2}\right)=1$. (Хотя кажется, что форма коллапсируюшего поля (6.1) выбрана достаточно произвольно, на самом деле эта форма подсказана компьютерным экспериментом по поиску минимума функционала массы (3.32). Далее мы увидим, что несмотря на простоту такой “анзац" в целом правильно воспроизводит основные свойства динамики коллапса.)

В дальнейшем мы будем интересоваться главным образом коллапсом классического кинка, т.е. решениями с $N_{1}=0$; этот процесс описывается семейством траекторий

$$
f(\xi, t)=f_{0}(\xi)+c(t) \Delta f(\xi)
$$

где

$$
\Delta f(\xi)=f_{N}(\xi)-f_{0}(\xi)
$$

является регулярной и (экспоненциально) убывающей на бесконечности функцией. Семейство параметризуется функцией $c(t)$ с граничными условиями

$$
c(0)=0, \quad \dot{c}(t)=0 ; \quad c(\infty)=1, \quad \dot{c}(\infty)=0 .
$$


Можно предположить (и это предположение будет подтверждено непосредственным вычислением), что процесс коллапса является адиабатическим в том смысле, что поле излучения можно рассматривать как малое возмущение в окрестности конфигурации $(6.2)$, а потери энергии на излучение за период колебаний переменной $c(t)$ малы по сравнению с самой энергией колебаний. Поэтому достаточно сначала рассмотреть динамику переменной $c(t)$ при произвольной (в пределах от начальной энергии кинка до нуля) энергии колебаний, а затем решить задачу на излучение для этой фоновой конфигурации.

Энергия конфигурации (6.2) вычисляется на основе формулы (3.32) с учетом дополнительных членов в производной по времени, содержащих $\dot{c}(t)$, что дает

$$
\begin{aligned}
M^{2}= & \frac{1}{2} \dot{c}^{2}(t) \int d \xi[\Delta f(\xi)]^{2}+ \\
& +\frac{1}{2} \frac{\partial}{\partial t}\left\{\dot{c}(t) \int d \xi\left[\left(1-\cos \hbar \partial_{\xi}\right) f(\xi, t)\right] \Delta f(\xi)\right\}+\int d \xi \mathcal{H}(\xi, t)
\end{aligned}
$$

где

$$
\mathcal{H}(\xi, t)=\frac{M^{2}}{2 \hbar^{2}}\left\{\left[\left(1-\cos \hbar \partial_{\xi}\right) f(\xi, t)\right]^{2}+\left[\left(\sin \hbar \partial_{\xi}\right) f(\xi, t)\right]^{2}\right\}+V(f(\xi, t)) .
$$

Заметим, что в соотношениях (6.5) и (6.6) функцию $f_{N}(\xi)$ без какой-либо сушественной потери точности можно заменить на $f_{\infty}(\xi)$, если $\hbar / N$ достаточно мало. Вычисление кинетического члена $T$, включаюшего операторы конечных разностей, в выражении для энергии $\int d \xi \mathcal{H}(\xi, t)$ можно провести, применив следующие соотношения, выведенные в [6]:

$$
\begin{aligned}
\left(\sin \hbar \partial_{\xi}\right) f_{N}(\xi) & =\frac{\hbar}{M_{N}} u^{\prime}\left(\alpha_{N} \xi\right)+O(\hbar), \\
\left(1-\cos \hbar \partial_{\xi}\right) f_{N}(\xi) & \simeq \frac{1}{2} \frac{\hbar^{2}}{M_{N}^{2}} u^{\prime \prime}\left(\alpha_{N} \xi\right)+O(\hbar),
\end{aligned}
$$

здесь штрих обозначает дифференцирование по аргументу. Поэтому при малых $\hbar$ член $\left[\left(1-\cos \hbar \partial_{\xi}\right) f_{N}\right]^{2}$ в выражении (6.6) для энергии много меньше члена $\left[\left(\sin \hbar \partial_{\xi}\right) f_{N}\right]^{2}$ и его можно отбросить. Таким образом, для кинетического члена в (6.5) получаем

$$
\int d \xi\left[\left(\sin \hbar \partial_{\xi}\right) f(\xi, t)\right]^{2}=\hbar^{2}\left(c_{0}^{2}+c_{N}^{2}\right)+2 \frac{\hbar^{2}}{M_{0} M_{N}} c_{0} c_{N} \int d \xi u^{\prime}\left(\alpha_{0} \xi\right) u^{\prime}\left(\alpha_{N} \xi\right) .
$$

Последнее слагаемое в $(6.8)$ имеет порядок малости $O\left(\hbar^{5 / 2}\right)$ и, следовательно, в $\sqrt{\hbar}$ меньше первого слагаемого, и его можно опустить. Поэтому окончательный ответ для кинетического члена приобретает вид

$$
T=\frac{M^{2}}{2}\left(c_{0}^{2}+c_{N}^{2}\right)=\frac{M^{2}}{2}\left[c^{2}+(1-c)^{2}\right] .
$$

Применяя ту же процедуру ко второму слагаемому в (6.5), можно показать, что оно имеет порядок малости $O\left(\hbar^{2}\right)$, т.е. в первом приближении им также можно пренебречь. 
Таким образом, в нулевом приближении выражение для энергии полевой конфигурации (6.2) может быть записано в виде

$$
M^{2}=\frac{\gamma}{2} \dot{c}^{2}+\frac{M^{2}}{2}\left[c^{2}+(1-c)^{2}\right]+W(c)
$$

где

$$
\gamma=\int d \xi[\Delta f(\xi)]^{2}, \quad W(c)=\int d \xi V\left[f_{0}(\xi)+c \Delta f(\xi)\right] .
$$

У кинкового коллапса существуют некоторые общие свойства, которые не зависят от конкретной полевой модели. Прежде всего $W(c=1)=0$, поэтому точка $c=1$ соответствует абсолютному минимуму энергии $W(c)$, в то время как $W(c=0)=M_{0}^{2} / 2$. Поэтому точка $c=1$ всегда лежит в классически разрешенной области, а скорость в этой точке окажется равной $\dot{c}^{2}=M^{2} / \gamma$.

В принципе возможны две ситуации: либо между точками $c=0$ и $c=1$ существуют классические траектории (нет потенциального барьера с энергией больше чем $W(c=0))$, либо барьер существует и тогда коллапс должен начинаться с туннелирования в окрестность точки $c=1$. В первом случае процесс коллапса будет состоять в периодическом движении между двумя точками поворота в потенциале $W(c)$, при этом на бесконечность будут излучаться волны (мы рассматриваем эти волны как возмушение, т.е. не учитываем их обратное влияние на динамику $c(t))$, которые будут уносить энергию, амплитуда колебаний будет убывать, а точки поворота - стремиться с двух сторон к точке $c=1$, соответствующей нулевой энергии колебаний, т.е. к предельной точке коллапса.

Во втором случае сначала должно иметь место туннелирование в классически разрешенную область, содержашую точку $c=1$, и лишь затем реализуется картина с затухающими колебаниями, описанная выше. Вероятность туннелирования может быть оценена в квазиклассическом приближении:

$$
P_{\text {tunn }} \simeq e^{-S / \hbar}
$$

где $S$ - классическое действие для траектории в запрешенной области.

Однако, по крайней мере в $(1+1)$-мерном случае для топологического кинка типа (4.5) всегда можно построить траекторию без потенциального барьера. Именно, рассмотрим семейство полевых конфигураций, удовлетворяющих следующим условиям: во-первых, они включают разрывное решение с монотонно возрастаюшей амплитудой; во-вторых, за исключением точки разрыва, значения поля стремятся к вакуумным; в-третьих, в точках, расположенных ближе к разрыву, стремление к вакуумному значению идет быстрее (тем самым производная по координате в каждой точке, кроме точки разрыва, убывает). Очевидно, что энергия таких конфигураций монотонно убывает по мере того, как полевая конфигурация стремится к $f_{\infty}(\xi)$.

Рассмотрим подробнее динамику коллапса в модели (4.4), аппроксимируя конечное состояния коллапса знаковой функцией $\operatorname{sign} \xi$. Вычисление интегралов в (6.11) дает

$$
\gamma=\frac{8}{3}(2 \ln 2-1), \quad W(c)=\frac{4}{3}(c-1)^{2}\left[\left(8 \ln 2-\frac{16}{3}\right) c^{2}+\frac{2}{3} c+\frac{2}{3}\right] .
$$




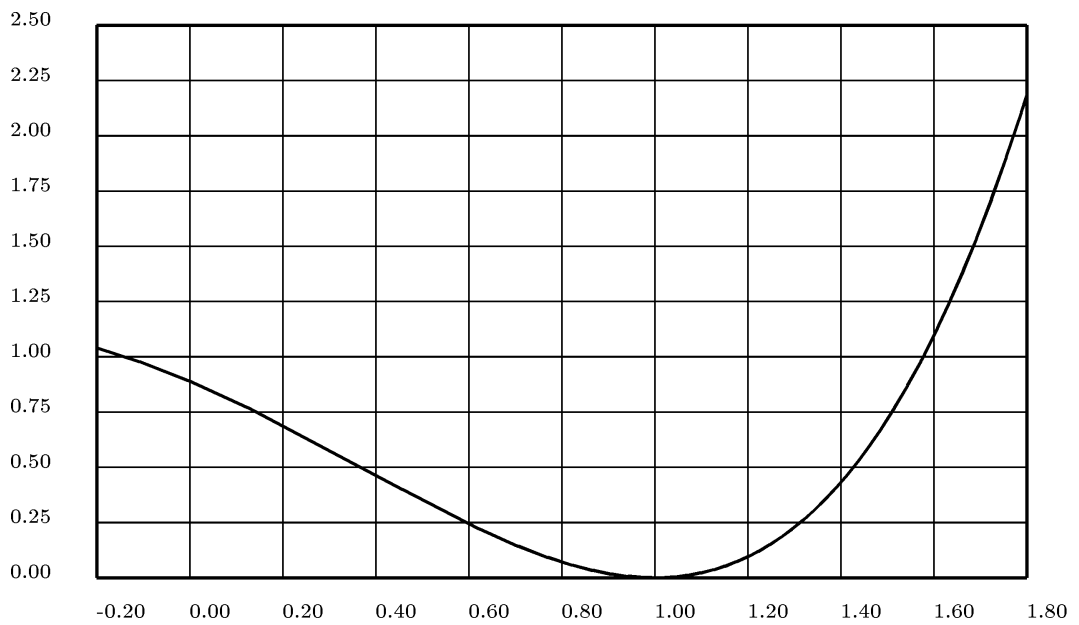

Рис. 1. раффик эффективного потенциала $W(c)$ для $\varphi^{4}$-модели.

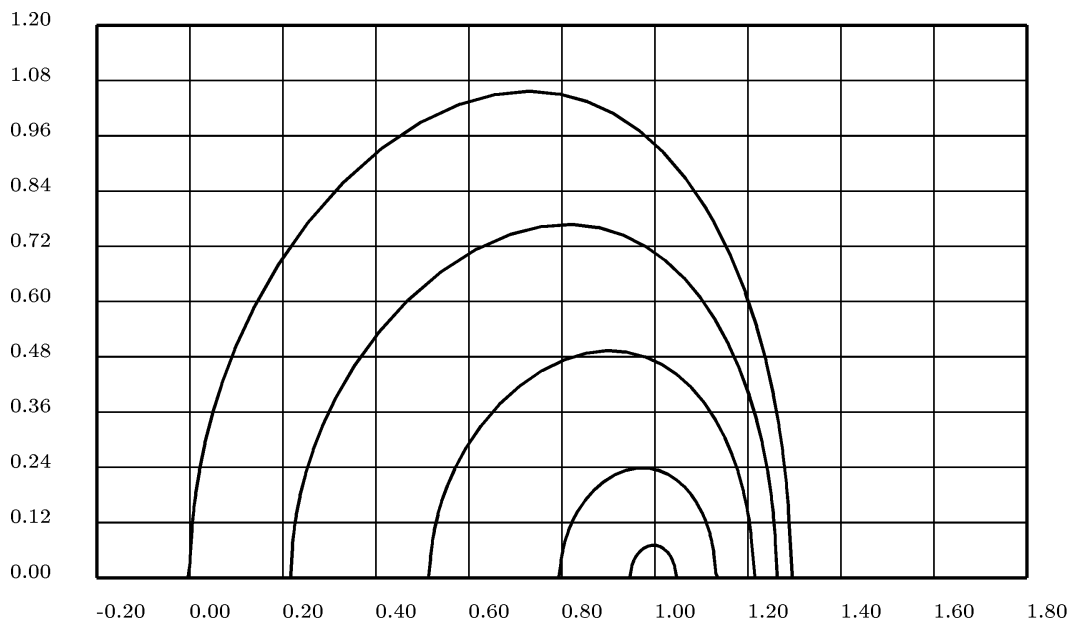

Рис. 2. Фазовые траектории $\dot{c}^{2}(t)$ для различных значений $M: M=4 / 3 ; 1,0 ; 2 / 3$; $1 / 3 ; 0,1$.

Из (6.13) следует, что если не учитывать потери энергии на излучение, динамика переменной $c(t)$ совпадает с динамикой нелинейного гармонического осциллятора, причем точки $c=0$ и $c=1$ не отделены друг от друга потенциальным барьером (рис. 1$)$. Следовательно, на начальных этапах коллапса (потери на излучение почти нулевые) $c(t)$ будет колебаться между точками $c_{1}=0$ и $c_{2} \simeq 1,2417$.

Потери энергии на излучение приведут к уменьшению амплитуды колебаний и соответственно к уменьшению массы $M$ кинкового решения. Фазовые траектории для различных значений $M$ приведены на рис. 2.

Для достаточно больших времен, когда $M$ стремится к нулю, уравнение колебаний в 
окрестности точки $c=1$ может быть линеаризовано:

$$
\frac{M^{2}}{2}=\frac{\gamma}{2} \dot{c}^{2}+W(c)
$$

Тогда, вводя обозначение $\widetilde{c}(t)$ :

$$
c(t)=1+\widetilde{c}(t),
$$

для отклонения от точки $c=1$ и полагая его малым, можно представить полевую конфигурацию при больших временах в виде

$$
f(\xi, t)=f_{\infty}(\xi)+\widetilde{c}(t) \Delta f(\xi) .
$$

Поскольку $f_{\infty}(\xi)$ - это почти разрывное решение, интерполируюшее различные вакуумные значения поля, то без какой-либо потери точности мы можем считать

$$
V\left(f_{\infty}\right)=V^{\prime}\left(f_{\infty}\right)=0
$$

Тем самым разложение энергии $W(c)$ по степеням $\widetilde{c}$ является по сути дела разложением в окрестности истинного статического вакуумного значения поля, т.е.

$$
\left.M^{2}\right|_{M \rightarrow 0}=\gamma\left[\dot{\tilde{c}}^{2}+m^{2} \widetilde{c}^{2}\right] .
$$

Следовательно, при асимптотически больших временах динамика $\widetilde{c}$ есть динамика гармонического осциллятора частоты

$$
\nu=m \text {. }
$$

Из (6.19), в частности, следует, что дискретные (вибрационные) моды в спектре возбуждения кинка $\phi_{n}(x)$ никак не участвуют в процессе коллапса. Это связано с тем, что дискретные моды всегда лежат ниже порога непрерывного спектра (массы мезона) $0<\omega_{n}<m$ и вероятность их генерации из-за несовпадения частот гораздо меньше, чем вероятность излучения уходящей на бесконечность волны. Эта ситуация вполне аналогична той, которая возникает в обычной квантово-механической теории рассеяния, где сушественно нелокальные (растущие на бесконечности) квазинормальные моды влияют на рассеяние, вызывая появление резонансов, а локализованные связанные состояния, которые в точности ортогональны состояниям рассеяния, никак в рассеянии не участвуют.

\section{7. АСИМПТОТИЧЕСКАЯ ДИНАМИКА КОЛЛАПСА В ОДНОМОДОВОМ ПРИБЛИЖЕНИИ}

Чтобы вычислить потери энергии системы на излучение, необходимо решить задачу об эволюции мезонной компоненты поля $\Phi(\xi, t)$ при наличии фоновой полевой конфигурации (6.2). Поскольку полная энергия поля сохраняется, то масса системы, состоящей из кинка и поля излучения, остается неизменной и равной $M_{0}$ в течение всего процесса 
коллапса. Вследствие этого в уравнениях движения после замены (2.7) даламбертиан превратится в оператор конечных разностей

$$
\square_{x t} \rightarrow \partial_{t}^{2}+2 \frac{M_{0}^{2}}{\hbar^{2}}\left(\cos \hbar \partial_{\xi}-1\right)
$$

Само же уравнение движения примет вид

$$
\left[\partial_{t}^{2}+2 \frac{M_{0}^{2}}{\hbar^{2}}\left(\cos \hbar \partial_{\xi}-1\right)\right] \widetilde{\varphi}(\xi, t)+V^{\prime}(\widetilde{\varphi}(\xi, t))=0
$$

здесь $\widetilde{\varphi}(\xi, t)$ обозначает поле в гейзенберговом представлении

$$
\varphi(x, t)=\widetilde{\varphi}(\xi(x, t), t)
$$

и является суммой кинковой и мезонной компонент

$$
\widetilde{\varphi}(\xi, t)=f(\xi, t)+\Phi(\xi, t)
$$

Поскольку классический кинк есть решение уравнений движения с точностью до $O(\hbar)$, то при $c \approx 0$ излучения практически нет, основной вклад в излучение дает окрестность точки $c=1$ (разумеется, речь идет о начальных стадиях коллапса, когда траектория $c(t)$ еще может находиться в окрестности $c \approx 0)$. При малых и промежуточных временах динамика системы может быть определена только численно. При больших временах эволюция $c(t)$ представляет собой линейные колебания в окрестности $c=1$, в этом случае можно получить аналитические оценки для динамики $c(t)$ и излучаемой энергии, поскольку излучение мало и поле излучения может рассматриваться как возмущение, т.е. процесс коллапса можно рассматривать в адиабатическом приближении: происходят колебания переменной $c(t)$ с энергией, медленно убываюшей за счет слабого излучения. Очевидно, что излучаемые волны будут иметь не квантовую, а классическую амплитуду, т.е. будут представлять собой когерентную волну. Кроме того, в рамках адиабатического подхода (энергия излучения мала) в спектре излучения не будут присутствовать моды с длиной волны порядка размера квантовой копии, следовательно, оператор конечных разностей для них можно заменить оператором дифференцирования:

$$
\left[\partial_{t}^{2}+2 \frac{M_{0}^{2}}{\hbar^{2}}\left(\cos \hbar \partial_{\xi}-1\right)\right] \Phi(\xi, t) \rightarrow \square_{y t} \widetilde{\Phi}(y, t)
$$

где $y=\alpha_{0} \xi$. Другими словами, наше описание излучения является в некотором смысле квазиклассическим.

Подставим теперь разложение (7.4) в (7.2) и линеаризуем его относительно $\Phi(\xi, t)$ в окрестности $f_{N}(\xi)$. Тогда уравнение для $\Phi(\xi, t)$ примет вид

$$
\left[\square_{y t}+V^{\prime \prime}\left(f_{N}\right)\right] \widetilde{\Phi}=j
$$


где правая часть (ток, вызывающий излучение) определяется невязкой в уравнении для $f(\xi, t)$ и явным образом зависит от $c(t)$ :

$$
-j(\xi, t)=\ddot{c}(t) \Delta f(\xi)+2 \frac{M_{0}^{2}}{\hbar^{2}}\left[\left(\cos \hbar \partial_{\xi}-1\right) f_{N}(\xi)\right]+V^{\prime}\left[f_{N}(\xi)\right]
$$

С помощью (6.7) оператор конечных разностей в (7.7) можно переписать в виде

$$
2 \frac{M_{0}^{2}}{\hbar^{2}}\left[\left(\cos \hbar \partial_{\xi}-1\right) f_{N}(\xi)\right] \simeq-\frac{M_{0}^{2}}{M_{N}^{2}} u^{\prime \prime}\left(\alpha_{N} \xi\right)
$$

Легко проверить, что при $\hbar / N \rightarrow 0$ имеет место соотношение

$$
\frac{M_{0}^{2}}{M_{N}^{2}} u^{\prime \prime}\left(\alpha_{N} \xi\right) \rightarrow \frac{Q M_{0}}{\alpha_{N}} \delta^{\prime}(\xi)
$$

где $Q$ - топологический заряд, которьй, разумеется, не зависит от $\hbar$. Множитель $\alpha_{N}^{-1}$ в правой части уравнения (7.9) стремится к нулю при $\hbar / N \rightarrow 0$, т.е. членом с конечными разностями в первом (квазиклассическом) приближении по $\hbar / N$ можно пренебречь.

Наконец, заменим в уравнении (7.6) функцию $f_{N}(\xi)$ на $f_{\infty}(\xi)$, для которой по тем же самым причинам, что и ранее, можно положить

$$
V^{\prime}\left(f_{\infty}\right)=0, \quad V^{\prime \prime}\left(f_{\infty}\right)=m^{2}
$$

Таким образом, уравнение движения для поля излучения можно записать в виде

$$
\left(\square_{y t}+m^{2}\right) \widetilde{\Phi}(y, t)=-\ddot{c}(t) \Delta f(\xi)
$$

Кроме того, чтобы избежать превышения точности, $\Delta f(\xi)$ можно заменить ее асимптотическим значением. Для рассматриваемой теории скалярного поля в $1+1$-измерениях типичным случаем является нечетный топологический кинк, тогда $\Delta f(\xi)$ можно представить в виде

$$
\Delta f(\xi) \simeq e^{-m|y|} \operatorname{sign} y, \quad y=\alpha_{0} \xi
$$

Теперь учтем, что при асимптотически больших временах колебания $c(t)$ становятся линейными с частотой $\nu=m$, т.е. положим

$$
c(t)=1+A \cos \nu t
$$

Решая уравнение (7.6) относительно $\Phi(\xi, t)$, легко вычислить энергию, излучаемую за период колебаний $c(t)$ :

$$
\Delta \mathcal{E}=4 \frac{\left(A \nu^{2}\right)^{2}}{\pi} \int \frac{d k}{k^{2}\left(k^{2}+m^{2}\right)} \sin ^{2}\left[\frac{\Delta t}{2} \sqrt{k^{2}+m^{2}}\right]=6.926 \frac{A^{2} \nu}{\pi}
$$


Следовательно, коллапсируюший кинк теряет за период энергию $\Delta \mathcal{E}=$ const $\times A^{2}$, где $A$ - текущая амплитуда колебаний величины $c$ в окрестности $c=1$. Поскольку энергия колебаний (т.е. остаточная масса коллапсирующего кинка) может быть оценена согласно (6.18) как

$$
M^{2}=\gamma \nu^{2} A^{2},
$$

то при достаточно больших временах эволюция коллапса описывается уравнением

$$
\frac{d M}{d t}=-\Delta \mathcal{E}=-C \times M^{2},
$$

где

$$
C=\frac{6.926}{\pi \nu \gamma} .
$$

Поэтому остаточная масса кинка $M(t)$ зависит от времени как

$$
M(t)=\frac{\text { const }}{t},
$$

а амплитуда колебаний в окрестности $c=1$ и интенсивность излучения убывают как $1 / t$ и $1 / t^{2}$, соответственно.

Из (7.18) следует вывод, что коллапс кинка может происходить только за бесконечное время, его закон является не экспоненшиальным, а полиномиальным. Следует подчеркнуть, что константа $C$ никакого физического смысла не имеет (она не является временем распада), это некоторый масштабный фактор размерности времени. Заметим также, что скорость распада не зависит от $\hbar$, поскольку роль квантовых эффектов в данном случае заключается только в том, что масса разрьвного решения оказывается нулевой, а сам процесс коллапса может быть описан в квазиклассических терминах.

Единственный случай, когда постоянная Планка будет влиять на динамику коллапса - это случай наличия потенциального барьера между начальным $(c=0)$ и конечным $(c=1)$ состояниями. Разумеется, $\hbar$ войдет в вероятность туннелирования

$$
P_{\text {coll }}=P_{\text {tunn }} \simeq e^{-S / \hbar},
$$

однако это будет не та постоянная (3.38), которая входит в операторы конечных разностей, а истинная "квантово-механическая" постоянная Планка. Заметим также, что зависимость от $\hbar$ через амплитуду туннелирования явно неаналитическая, что еше раз подчеркивает непертурбативную природу эффекта.

В отсутствие барьера (как это, по-видимому, имеет место для всех одномерных топологических кинков)

$$
P_{\text {coll }}=1 .
$$

Разумеется, асимптотическая динамика при больших временах не зависит от наличия или отсутствия барьера, и в обоих случаях закон коллапса будет полиномиальным. 


\section{8. РЕЗУЛЬТАТЫ ЧИСЛЕННОГО АНАЛИЗА ДИНАМИКИ КОЛЛАПСА}

В этом разделе изложены результаты численного анализа динамики коллапса в $\varphi^{4}$-модели (4.4). Вычисления проводились двумя различными методами: первый состоит в рассмотрении динамики переменной $c(t)$, эволюционирующей в потенциале $W(c)$ при убываюшей энергии. Мезонное поле излучения определяется посредством численного решения точного (нелинейного) уравнения движения для $\Phi(\xi, t)$ на фоне коллапсируюшего кинка $f(\xi, t)=f_{0}(\xi)+c(t) \Delta f(\xi)$. В динамическом уравнении $(6.10)$ для $c(t)$ текушее значение массы $M(t)$ определяется из закона сохранения энергии путем вычисления полной энергии, излученной коллапсируюшим кинком на бесконечность к данному моменту времени. Вычисления проводились стандартным методом Рунге-Кутта. Графики полного поля $\varphi(\xi, t)$ (сумма фонового поля $f(\xi, t)=f_{0}(\xi)+c(t) \Delta f(\xi)$ и поля мезонного излучения $\Phi(\xi, t))$ для некоторых моментов времени приведены на рис. 3 .

Видно, что амплитуда осцилляций поля $f(\xi, t)$ убывает со временем, интенсивность излучения также убывает, а длина излучаемой волны растет. Однако в рамках этого метода в принципе невозможно достичь "настоящего" длинноволнового предела (хотя частота колебаний и стремится к $m=2$, соответствуюшей бесконечно большой длине волны). Это связано с тем, что в фоновое поле (входяшее как источник в правую часть уравнений для $\Phi(\xi, t))$ изначально заложен пространственный масштаб - размер классического кинка. Более того, эволюция поля при этом подходе является в некотором смысле нелокальной, динамика $c(t)$ определяется посредством интегрирования по расстояниям порядка размера кинка, а текушее значение энергии колебаний $M(t)$ находится суммированием энергии, излученной в предыдушие моменты времени. Поэтому динамика $c(t)$ постоянно "запаздывает" по сравнению с динамикой $\Phi(\xi, t)$. Тот факт, что эволюция $\Phi(\xi, t)$ сушественно определяется эволюцией $c(t)$, делает это запаздывание еше больше. На рис. 3 хорошо заметен (отрицательный) сдвиг фазы осцилляций на левом конце кривой по сравнению с осцилляциями посередине.

Таким образом, численное моделирование процесса коллапса посредством однопараметрической модели, хотя и позволяет качественно воспроизвести его основные свойства, обладает рядом слабых мест и вполне удовлетворительным не является. Альтернативный подход заключается в следуюшем. Рассмотрим классическое уравнение поля

$$
\square_{x t} \varphi(x, t)+V^{\prime}(\varphi(x, t))=0
$$

и модифишируем его таким образом, чтобы оно допускало не только решение в виде обычного кинка, но и другое решение практически нулевого размера. Это может быть достигнуто путем введения демпфируюшей функции

$$
\Lambda_{\varepsilon}(x)=\varepsilon^{2}+\left(1-\varepsilon^{2}\right) \operatorname{th}^{2} \lambda x
$$

в слагаемое с производными по пространственному аргументу поля. Уравнение движения тогда примет вид

$$
\left[\partial_{t}^{2}-\Lambda_{\varepsilon}(x) \partial_{x}^{2}\right] \varphi(x, t)+V^{\prime}(\varphi(x, t))=0
$$

3 Теоретическая и математическая физика, т. 108, № 2, 1996 г. 

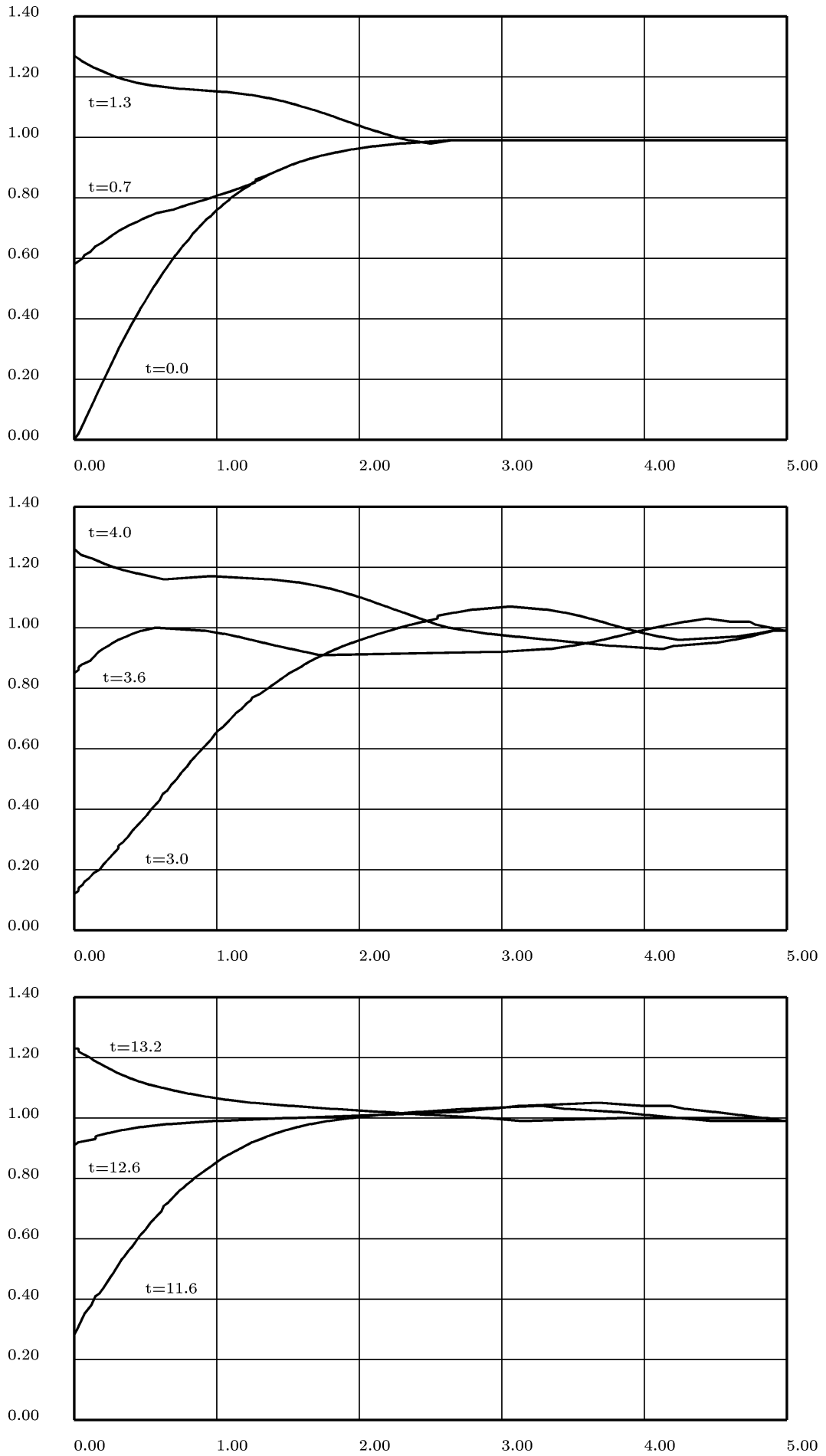

Рис. 3. Граффики поля $\widetilde{\varphi}(\xi, t)$ в разные моменты времени. 
Если выбрать параметры $\varepsilon$ и $\lambda$ в функции $\Lambda_{\varepsilon}(x)$ таким образом, что

$$
1 / \varepsilon \gg \lambda \gg 1
$$

то уравнение (8.3) будет допускать два статических решения - обычный кинк с размером $O(1)$ и его копию размером $O(\varepsilon)$. Энергия копии оказывается порядка $O(\varepsilon)$, т.е. следующего порядка малости по сравнению с "обычным" кинком. Следовательно, введение демпфирующей функции достаточно удачно моделирует появление квантовых копий.

Динамика кинкового коллапса теперь может быть моделирована путем численного решения уравнения (8.3) с начальным условием, соответствующим статическому классическому кинку. Решение также проводилось методом Рунге-Кутта. Результаты вычислений представлены на рис. 4.

Необходимо отметить следующие особенности процесса коллапса: во-первых, в отличие от результатов, полученных предыдушим методом, при малых временах классический кинк (который является точным статическим решением) не эволюционирует, пока его не достигнет возмущение, распространяюшееся из окрестности нуля; во-вторых, при больших временах динамика поля выходит на "настоящий” длинноволновой предел, который также не достигался при предыдушем подходе.

Графики зависимости $M(t)$, вычисленные обоими методами, приведены на рис. 5 . Плато на начальном интервале соответствует временам, когда излучение еше не достигает энергетической ловушки, которая изображает пространственную бесконечность. Видно, что на качественном уровне динамика коллапса в обоих случаях имеет один и тот же характер, но при этом однопараметрический (первый) метод занижает излучение на начальной стадии коллапса. К сожалению, мы не можем утверждать, что достигли по-настоящему больших времен, при которых динамика описывается степенным законом (7.18).

На первом графике можно наблюдать детали зависимости энергии от времени на начальных стадиях коллапса, второй график соответствует относительно большим временам. Видно, что при достаточно больших временах кривая, полученная однопараметрическим методом, соответствует большим потерям энергии по сравнению с кривой, полученной вторым методом. Это связано с тем, что источник, появляюшийся в правой части уравнений движения при использовании первого метода, явным образом содержит пространственный масштаб (размер кинка), что стимулирует излучение. При вычислении вторым методом такой стимулящии не происходит, так что при больших временах реализуется правильный длинноволновой предел.

K сожалению, вычисления предъявляют довольно большие требования как к памяти, так и к быстродействию вычислительной техники (несмотря на то что как память, так и время вычислений растут не более чем линейно с ростом длины рассматриваемого пространственного отрезка $L$ ). При вычислениях необходимо делать $L$ достаточно большим, чтобы увеличить расстояние от коллапсируюшего кинка до "пространственной бесконечности" (точки, в которой помешена энергетическая ловушка): чем больше это расстояние, тем более мы приблизимся к длинноволновому пределу. Действительно, при длине волны, сравнимой с $L$, мы получаем не волны, излучаемые на бесконечность, а стоячую волну с волновым числом $\pi / 2 L$, узел которой приходится как раз на 

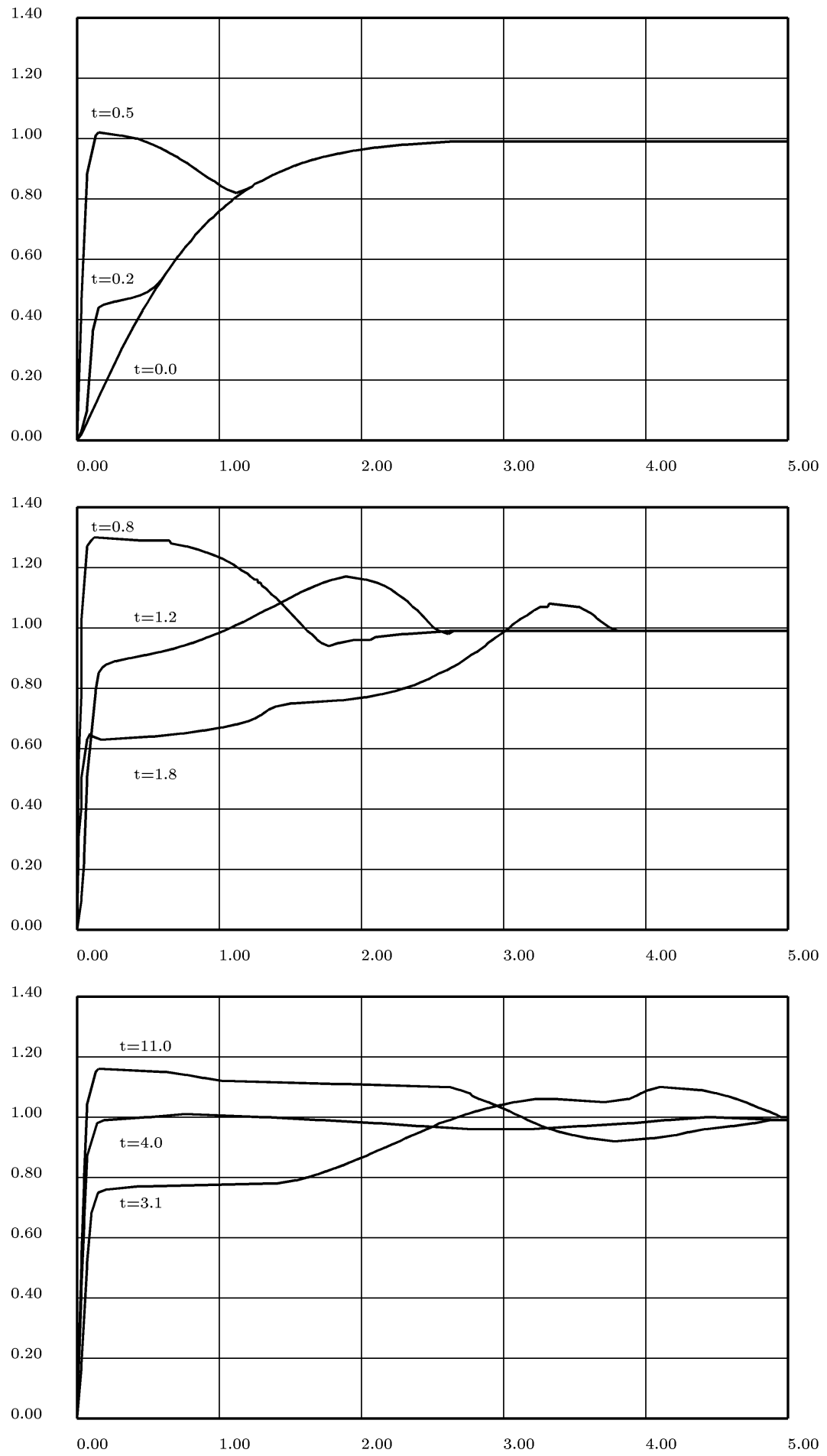

Рис. 4. Динамика коллапса $\varphi^{4}$-кинка, полученная путем решения уравнения (8.3) в разные моменты времени. 

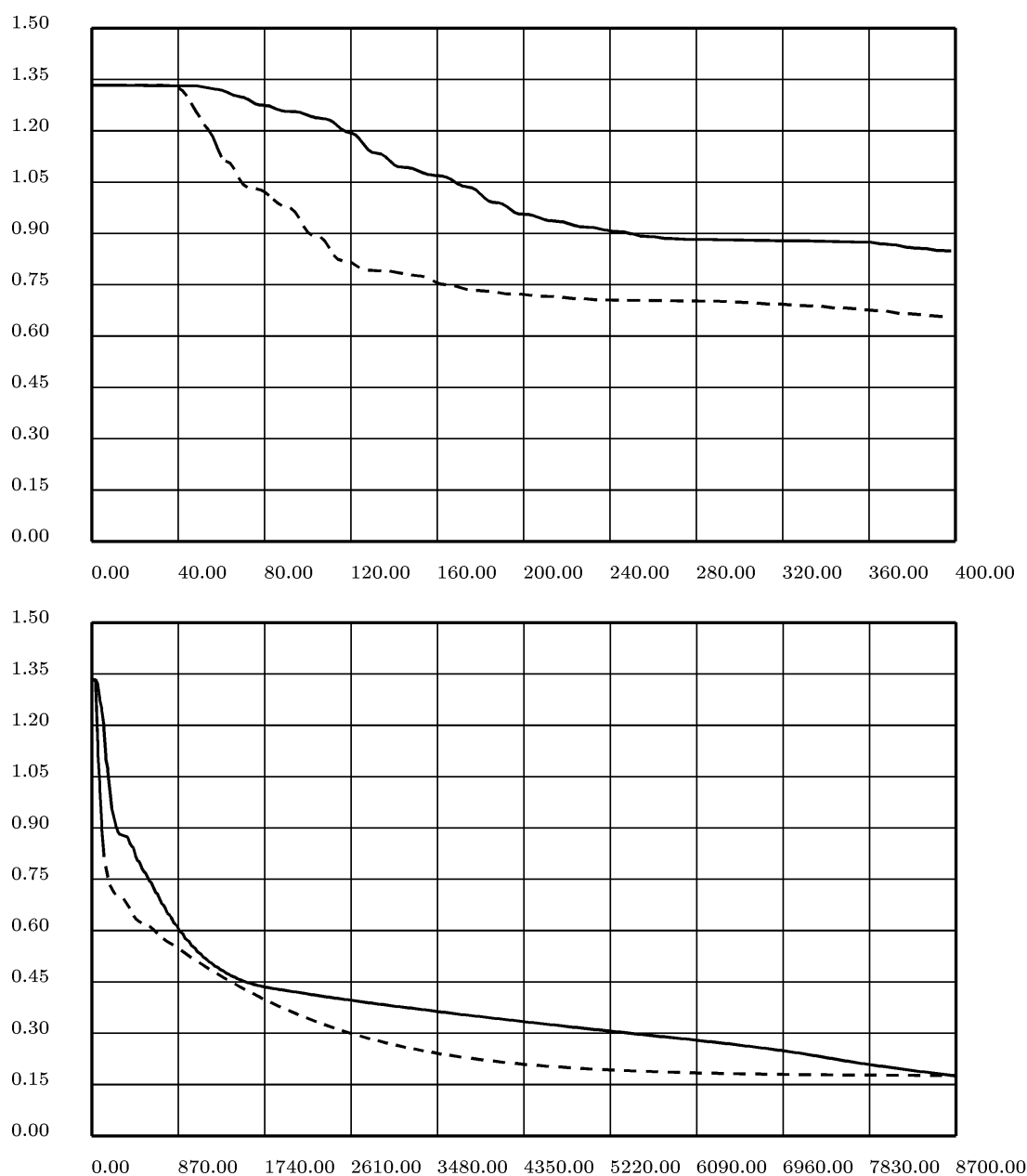

Рис. 5. График убывания массы кинка при коллапсе в $\varphi^{4}$-модели. Сплошная кривая соответствует вычислениям, выполненным с использованием (6.10), а штриховая - с использованием (8.3).

энергетическую ловушку (именно при больших временах поле $\widetilde{\varphi}(\xi, t)$ и приобретает такую форму), так что поглошение энергии почти полностью прекрашается и расчеты перестают описьвать реальную картину коллапса.

Нам удалось достичь такой комбинации времени вычислений и длины интервала $L$, при которой оказывается излученной около $90 \%$ от начальной массы кинка $M_{0}=4 / 3$. При этом результаты еше не позволяют сделать однозначное заключение о том, достигли ли мы асимтотического полиномиального поведения или еше нет.

\section{9. ЗАКЛЮЧЕНИЕ}

Таким образом, при такой непертурбативной процедуре вычитания расходимостей посредством вычеркивания полюсных членов из производных поля, основанного на 
уравнении (3.13), удается построить вполне содержательную схему лоренц-ковариантного квантования кинков, в которой конечные разрывы полевой конфигурации не дают расходимостей, а напротив, их энергия убывает с уменшшением переходной области разрыва. Несмотря на нетривиальность результата изложенный подход основывается, по существу, на следующих достаточно очевидных допущениях: 1) должно выполняться условие ковариантности квантованной теории относительно группы Пуанкаре (1.1), т.е. при разложении поля в окрестности кинка мы обязаны потребовать, чтобы это условие выполнялось по отдельности для кинковой и мезонной компонент; 2) для таких локализованных объектов, как кинки, должна быть ковариантным образом определена координата центра масс; 3) расходимость на конечном разрыве полевой конфигурации обусловлена высокочастотной компонентой поля, поэтому ультрафиолетовое обрезание теории должно сглаживать такие сингулярности. Условия 1 и 2 однозначным образом приводят нас к замене (2.4), а в совокупности с условием 3 дают разностные уравнения (3.7) и (3.32) на профильную функцию и массу кинка. В свою очередь, эти уравнения обеспечивают "регуляризацию" (и более того, равенство нулю) массы предельной кинковой копии $f_{\infty}(\xi)$. В результате даже при наличии локальной устойчивости относительно малых флуктуаций становится возможен бесконечно медленный (полиномиальный) коллапс кинка в состояние с почти нулевыми массой и размерами, но тем же топологическом зарядом. Еще раз подчеркнем, что как сама разностная структура, так и последний эффект являются сушественно непертурбативными, поскольку как подстановка (2.4)-(2.7), так и кинковые решения, а тем самым и их полюсы, и вычеты $a_{n}$ и $\Gamma_{n}$, являются результатом частичного суммирования ряда теории возмушений. С другой стороны, именно это обстоятельство и обусловливает невозможность установить детальное соответствие между полюсными членами и стандартными ультрафиолетовыми расходимостями диаграммной теории возмущений, поскольку для этого требуется суммирование всего ряда теории возмушений, которого, как известно, может и не быть [27]. Поэтому нельзя утверждать, что рассмотренное нами вычитание полюсных членов не означает попытку придать смысл квантовой теории там, где стандартные методы могут просто не работать.

В настоящей статье мы ограничились рассмотрением $(1+1)$-мерного случая. Однако можно показать [28], что и в большем количестве измерений требование явной ковариантности приводит к операторам конечных разностей в уравнениях движения. Эти операторы по форме аналогичны операторам конечных разностей на массовой поверхности в рамках квазипотенциального подхода [29], чем еше раз подтверждается естественность наших построений при лоренш-ковариантном подходе к квантованию кинков. Следовательно, можно ожидать, что и в многомерном случае должны проявиться эффекты, аналогичные вышеизложенным.

Необходимо также отметить, что из нашего анализа отнюдь не следует, что такой коллапс является неизбежным концом эволюции любого кинкового состояния. Действительно, в нашем подходе мы, по сушеству, рассматриваем динамику мезонной компоненты $\Phi(\xi, t)$ чисто пертурбативно, полностью игнорируя обратное влияние излучения на кинковую составляющую. Это, однако, является допущением, которое может и не выполняться из-за нелинейных эффектов. Вопрос о том, когда это можно делать, зависит от специфики конкретной модели. Примером, когда этого делать нельзя, является 
модель Sine-Gordon (SG), где за счет бесконечного набора динамических законов сохранения, которые справедливы и в квантовом случае, излучение мезонов SG-солитонами невозможно. Это же подтверждается и квантовым методом обратной задачи рассеяния [30], из которой следует, что в модели SG солитонное состояние в точном решении является стабильным.

С другой стороны, гипотеза о возможности коллапсирования кинков никоим образом не входит в противоречие с реальной ситуацией в физических приложениях, поскольку речь может идти только о релятивистских квантово-полевых моделях в плоском пространстве-времени. Таким образом, сразу отпадают все кинковые возбуждения в конденсированных средах, поскольку в последнем случае исходная система является решеточной и тем самым заведомо нарушает лоренц-инвариантность. Поэтому, даже если эффективная непрерывная теория, полученная из исходного решеточного гамильтониана, и будет иметь формально ковариантньй квантово-полевой вид, то эта симметрия фундаментальной не является, и условие ковариантности (1.1) в данном случае неприменимо. Поэтому остаются лиш квантово-полевые модели в физике частиц, где реальных кандидатов всего два - это калибровочные теории Янга-Миллса-Хиггса с неабелевыми магнитными монополями [1] и скирмионные модели барионов [31-33]. Однако экспериментальных указаний на существование магнитных монополей до сих пор нет, в то время как во втором случае статус модели Скирма также неясен, поскольку она является неперенормируемой и может рассматриваться лишь как некоторая эффективная квазиклассическая теория. Кроме того, исходная киральная сигма-модель без скирмовского члена уже сама по себе нестабильна относительно коллапса, и существует ряд гипотез, что эта нестабильность может быть преодолена на квантовом уровне [34-36]. Эта проблема в рамках нашего подхода будет рассмотрена отдельно.

Работа была выполнена при частичной финансовой поддержке Российского фонда фундаментальных исследований по грантам № 94-02-05490/91 и 96-02-18097, Санкт-Петербургского конкурсного центра фундаментального естествознания и научной программы "Российские университеты".

\section{Список литературы}

[1] R.Rajaraman. Solitons and Instantons. Amsterdam: North-Holland, 1982.

[2] Chiral Solitons / Ed. K.F.Liu. Singapoure: World Scientific, 1987.

[3] L.Wilets. Nontopological Solitons. Singapoure: World Scientific, 1989.

[4] T.D.Newton, E.P.Wigner // Rev. Mod. Phys. 1949. V. 21. P. 400.

[5] G.Semenoff, H.Matsumoto, H.Umezawa // J. Math. Phys. 1981. V. 22. P. 2208; // Progr. Theor. Phys. 1982. V. 67. P. 1619.

[6] K.Sveshnikov // Nucl. Phys. 1993. V. B405. P. 451; // Int. J. Mod. Phys. 1994. V. A9. P. 2586.

[7] J.-L.Gervais, A.Jevicki, B.Sakita // Phys. Rev. 1975. V. D12. P. 1038.

[8] E.Tomboulis // Phys. Rev. 1975. V. D12. P. 1678.

[9] N.-H.Christ, T.D.Lee // Phys. Rev. 1975. V. D12. P. 1606.

[10] A.Hosoya, T.Kikkawa // Nucl. Phys. 1975. V. B101. P. 271.

[11] E.Tomboulis, T.T.Woo // Ann. of Phys. (N.Y.) 1976. V. 98. P. 1.

[12] X.Yang, J.A.Parmentola // Mod. Phys. Lett. 1989. V. A4. P. 2509.

[13] P.Jain // Phys. Rev. 1990. V. D41. P. 3273; // Mod. Phys. Lett. 1991. V. A6. P. 2366.

[14] J.A.Parmentola, X.Yang, I.Zahed // Ann. of Phys. (N.Y) 1991. V. 209. P. 127. 
[15] P.Jain // Phys. Rev. 1991. V. D44. P. 3982.

[16] O.Steinmann // Nucl. Phys. 1977. V. B131. P. 459; 1978. V. B145. P. 141.

[17] H.Matsumoto, N.J.Papastamatiou, H.Umezawa, M.Umezawa // Phys. Rev. 1981. V. D23. P. 1339.

[18] H.Matsumoto, N.J.Papastamatiou, G.Semenoff, H.Umezawa // Phys. Rev. 1981. V. D24. P. 406.

[19] К.А.Свешников // ТМФ. 1983. Т. 55. №3. С. 361-384; 1988. Т. 74. №3. С. 373-391.

[20] О.А.Хрусталев, К.А.Свешников // ТМФ. 1992. Т. 93. № 3. С. 384-402.

[21] В.Б.Тверской // ТМФ. 1986. Т. 68. № 3. С. 338-349.

[22] A.Calogeracos // Phys. Rev. 1990. V. D42. P. 573.

[23] F.Aldabe, D.R.Bess, N.N.Scoccola // Phys. Lett. 1992. V. B293. P. 81; 1993. V. B304. P. 98.

[24] J.P.Garrahan, M. Kruczenski, G.L. Schat, D.R.Bess, N.N.Scoccola // Phys. Rev. 1995. V. D51. P. 2950.

[25] М.А.Лаврентьев, Б.В.Шабат. Методы теории функций комплексного переменного. М.: ГИТТЛ, 1958.

[26] J.Goldstone, R.Jackiw // Phys. Rev. 1975. V. D11. P. 1486.

[27] G. t'Hooft // The Whys of Subnuclear Physics. N.Y.: Plenum Press, 1979.

[28] K.Sveshnikov // Phys. Lett. 1989. V. A136. P. 1; // TMФ. 1990. T. 82. № 1. C. 55-65.

[29] В.Г.Кадышевский, З.М.Мир-Касимов, Н.Б.Скачков // ЭЧАЯ. 1972. Т. 2. С. 637.

[30] Е.К.Склянин, Л.А.Тахтаджиян, Л.Д.Фаддеев // ТМФ. 1979. Т. 40. №2. С. 194-220.

[31] I.Zahed, G.E.Brown // Phys. Rep. 1986. V. 142. P. 1.

[32] G.Holzwarth, B.Schwesinger // Rep. Progr. Phys. 1986. V. 49. P. 825.

[33] В.Г.Маханьков, Ю.П.Рьбаков, В.И.Санюк // УФН. 1992. Т. 162. С. 1.

[34] P.Jain, J.Schechter, R.Sorkin // Phys. Rev. 1989. V. D39. P. 998; 1990. V. 41. P. 3855.

[35] J.A.Mignaco, S. Wulck // Phys. Rev. Lett. 1989. V. 62. P. 1449.

[36] N.Chepilko, K.Fujii, A.Kobushkin // Phys. Rev. 1991. V. D43. P. 2391; V. 44. P. 3249.

Физический факультет и

Поступила в редакцию

Институт теоретических проблем микромира

17.II.1995 г.

Московского государственного университета

\section{K.A. Sveshnikov, P.K. Silaev \\ CONNECTION BETWEEN DISCONTINUOUS STEP-LIKE AND SMOOTH KINK-TYPE CLASSICAL SOLUTIONS IN QUANTUM FIELD THEORY}

Non-perturbative procedure for subtraction of singularities, caused by discontinuous step-like solutions in nonlinear QFT is suggested. We discuss in details the procedure of Lorentz-covariant quantization for topological kinks in $(1+1)$ nonlinear field models. It had been shown that the "quantum copies" of classical kink appears as the result of this procedure. These copies have the same topological charge, but negligibly small size. Moreover, they have negligibly small mass, due to subtraction procedure, that eliminate divergent terms that are proportional to squared delta-function. We give the detailed discussion of these problems for various $(1+1)$ nonlinear models with classical kinks. 